\title{
Final report on the Magnetized Target Fusion Collaboration
}

\author{
DOE Award: DE-FG02-04ER54753 \\ UW BN 62-9935
}

\author{
Prof. John Slough, P.I.
}

\section{Plasma Dynamics Laboratory}

Department of Aeronautics and Astronautics

Box 352400

University of Washington

Seattle, WA 98195 


\section{Preface to the final report}

The final report presented here represents the work performed in the first phase of the Magnetized Target Fusion (MTF) collaboration. The work was shifted from the University of Washington to a private company - MSNW LLC. The reason for this was the lack of sufficient space and personnel to continue the work at the University of Washington. More critically, the experimental apparatus on which the future work was to be performed was built and operated by MSNW. Initially the device (the Inductive Plasma Accelerator or IPA) was to be constructed at the UW. As mentioned, there was no adequate space available at the UW campus, and it was decided by the Aeronautics and Astronautics Department to transfer the experiment to MSNW facilities for all future work on IPA. It was therefore necessary to transfer the continuing work on MTF to MSNW as well. The report therefore covers the initial work performed at the Plasma Dynamics Laboratory at the University of Washington, as a discussion of the extension of this work to be performed at MSNW. 


\section{Final report on the Magnetized Target Fusion Collaboration}

A promising approach to compression of the FRC for small scale fusion, employs a metal shell to radially compress the FRC plasmoid. This approach has been under study now for several years and is referred to as the Magnetized Target Fusion (MTF) concept. In this approach, the presence of the magnetic field in the target plasma suppresses the thermal transport to the confining shell, thus lowering the imploding power needed to compress the target to fusion conditions. With MTF the energy needed to heat and compress the FRC to fusion conditions is provided by the implosion of a metal liner.

It is critical that the target plasma for compression satisfy certain conditions if the subsequent liner compression is to achieve breakeven or better conditions. The achievement of these target parameters are in themselves a considerable challenge, and one that has been occupying the MTF group at the Los Alamos National Laboratory (LANL) for the past several years. The primary and single most important aspect of the research to be performed was therefore to:

Provide a robust, efficient, and expedient method of FRC formation, acceleration and compression to achieve a suitable target for attaining fusion conditions.

The Magnetize Target Fusion (MTF) experiments at Los Alamos National Laboratory (LANL) and the Air Force Research Laboratory (AFRL) represent an attempt to compress plasmas to fusion conditions at the highest densities for a magnetically confined plasma. The experiment to be performed is a very ambitious one that would amount to a major advance if successful. However, it is not a trivial experiment, and there is little prior history of plasmoid compression experiments to draw experience or scaling from. Recent progress and breakthroughs achieved at MSNW employing a novel plasma formation acceleration scheme referred to as the Inductive Plasmoid Accelerator provide for a viable method for achieving the target parameters for the $\mathrm{Q}=1$ metal liner compression experiments at the Air Force Research Laboratory, as well as provide for a test bed for characterization and optimization of the concept. The application of the IPA as a test bed facility for studying the merging of FRCs and subsequent liner compression provide a low cost method for exploring both an alternate approach to target plasma formation (the merging experiments), as well as provide a facility that could be used to study liner/plasma dynamics and interaction with a much lower liner energy. It would not be difficult to further extend the IPA device to study the implosion of metallic foil liners on FRC plasmoids as well. The energy requirement for foil implosions to the $100 \mathrm{~T}$ level would be in the $100 \mathrm{~kJ}$ range. The destructive aspects from imploding a metallic liner as massive as that planned for the MTF experiment at AFRL could be avoided. This would allow for greater diagnostic access, and more extensive testing.

These alternate methods would operate in a manner that doesn't require the large investment in pulse power and equipment required for the massive liner implosion. One could then perform detailed physics study of the compression process starting initially with a simple compression coil. These experiments could be followed by compression with a plasma liner progressing to a foil liner. Both of these approaches would allow for 
detailed study of liner compression without the destructive aspects of the massive liner implosion to be employed in the AFRL experiments.

An experimental demonstration of the acceleration/compression of the FRC plasmoid to the conditions listed in Table I is the primary task to be carried out.

This undertaking will take advantage of a simple and direct approach that has recently proven to provide a stable, long-lived, hot FRC of appropriate flux and inventory. Based on these results it is believed that with straight forward modification of the existing IPA apparatus, along with the dynamic formation /acceleration technique that was utilized in the IPA experiments, the resulting FRC can achieve the parameters far in excess of those presented in Table I for MTF fusion.

The actual start of the effort was delayed until the receipt of funding in at MSNW in October of 2007 so that the work described here reflects the final work at the UW as basically the

\begin{tabular}{ll}
\hline \hline Parameter & Design spec. \\
\hline coil $E_{\theta}(\mathrm{kV} / \mathrm{cm})$ & 1 \\
coil radius $(\mathrm{cm})$ & 5.0 \\
separatrix radius $(\mathrm{cm})$ & 2.5 \\
coil length $(\mathrm{cm})$ & 36 \\
separatrix length $(\mathrm{cm})$ & 35 \\
$B_{\text {ext }}(\mathrm{T})$ & 5 \\
$B_{\text {bias }}(\mathrm{kG})$ & 4 \\
$B_{\mathrm{GN}}(\mathrm{kG})$ & 6.6 \\
$p_{0}$ gas fill $(\mathrm{mTorr})$ & 80 \\
peak density $\left(10^{17} \mathrm{~cm}^{-3}\right)$ & 1.0 \\
$T_{\mathrm{e}}+T_{\mathrm{i}}(\mathrm{keV})$ & 0.6 \\
plasma energy $(\mathrm{kJ})$ & 7 \\
$\tau_{\mathrm{N}}(\mu \mathrm{s})$ & 28 \\
particle inventory $\left(10^{19}\right)$ & 5 \\
$\Phi_{\text {bias }}(\mathrm{mWb})$ & 4 \\
$\Phi_{\mathrm{LO}}(\mathrm{mWb})$ & 4 \\
$\Phi_{\text {int }}$ internal flux $(\mathrm{mWb})$ & 1.0 \\
$S^{*}$ & 25 \\
$s$ & 2.9 \\
$E$ & 7 \\
$S^{*} / E$ & 3.5 \\
\hline \hline
\end{tabular}

Table I. FRC Parameters for initial target plasma for MTF starting phase of the project at MSNW. During the first phase, the design and testing of the key components for the formation, acceleration and compression were to be performed, and these tasks have already been successfully completed. In addition, both numerical and analytical work to optimize the compression has been carried out and will be described later. The upgrade to the existing IPA experiment has also been completed. A discussion of theses changes will be discussed in detail in this report. 
The target formation method will be the same as that on IPA and has been described elsewhere. However it deserves to be pointed out in that it is what makes this particular approach so effective, i.e. the energy that is required to achieve the high density and temperature of the initial target FRC is derived from axial kinetic energy of the FRC's motion prior to compression. The simultaneous formation, acceleration and merging of two FRCs results in the most rapid method for assembling a stationary target for compression. By starting the FRCs in a larger radius formation/accelerator the

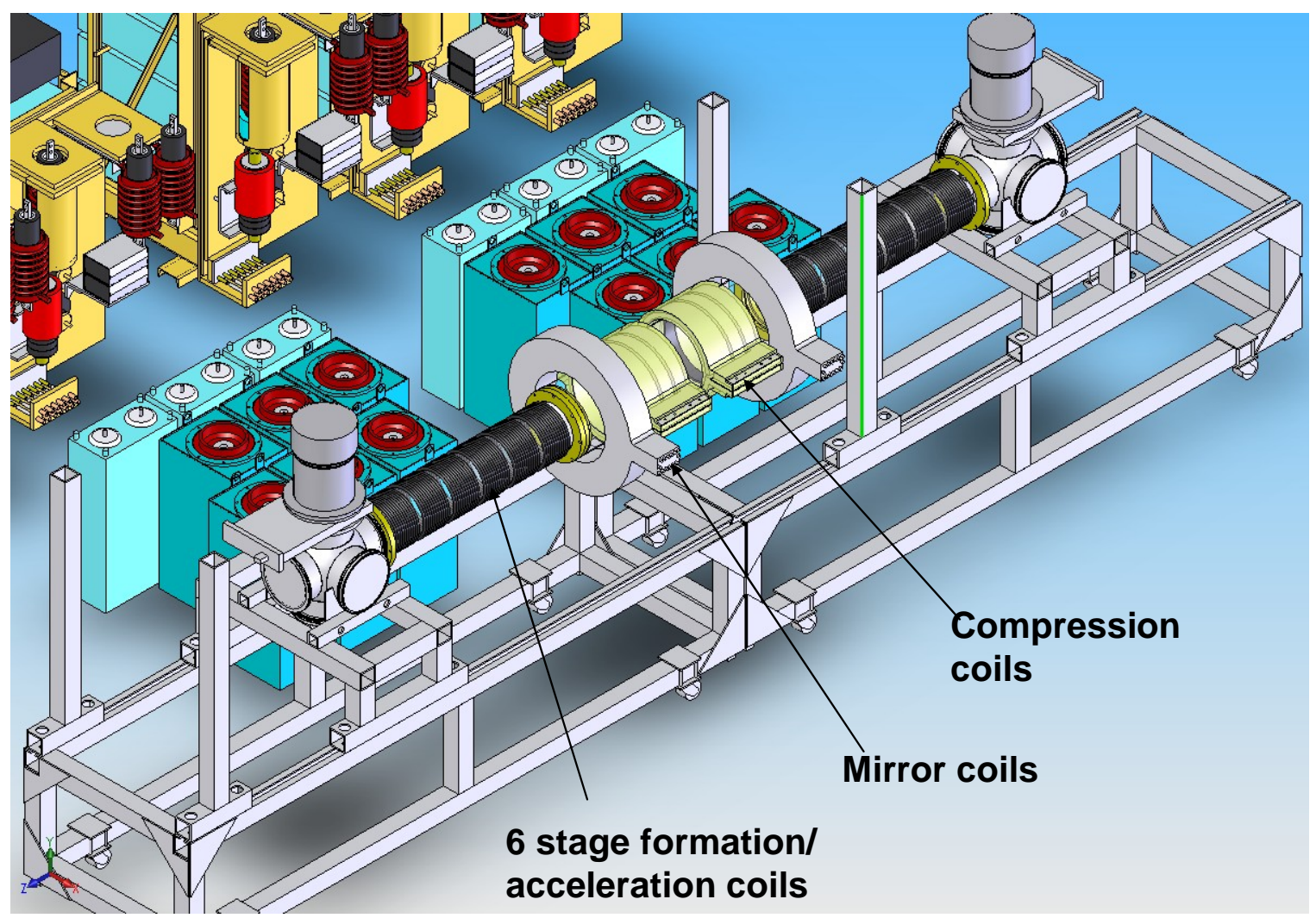

Figure 1. Arrangement of the two Inductive Plasma Accelerators used in the initial compression experiments.

achievement of the appropriate inventory and flux is readily accomplished (as it was in the initial IPA experiments). The configuration best suited for the initial experiments will continue to employ both IPAs aligned in opposition (see Fig. 1) A high density FRC plasmoid is formed and accelerated out of each IPA into a merging/compression chamber. The colliding/merging is then compressed by a final coil to achieve a size suitable for injection into the metal liner. In this manner both a staged axial and radial compression of two FRCs will be realized. A discussion of the IPA facility and a brief description of the results will be given in the next section. A discussion of the new design will be given along with results from numerical calculations. The new device is referred to as IPA-C. 


\section{MTF Target Formation using the IPA-C Facility}

\section{Results from the IPA Experiments}

In the Inductive Plasma accelerator (IPA) the acceleration of the FRC plasmoid is through the electromagnetic interaction of the radial magnetic field of the sequentially activated accelerator coils (see Fig. 1), and the large FRC toroidal plasma current through the Lorentz force. The electrical efficiency of such an accelerator can be quite high (>90\%) if active switching is employed. In the repetitive mode of operation, any energy that is not imparted to the FRC by each accelerator coil is returned to the capacitor for the next pulse. The only significant mechanism for energy loss is the circuit resistance, which can be kept low compared to the kinetic energy imparted to the FRC. The energy storage requirements for each FRC accelerator are low and the charge voltage is in the conventional range for thyratron switched pulsed power. The magnetic forces on the coils are also small. The magneto-kinetic compression heating of the FRC plasma has the potential to be much more efficient than other methods. Not only is the energy coupling mechanism low loss, virtually all of the kinetic energy is thermalized in the plasma in the form of ion energy.

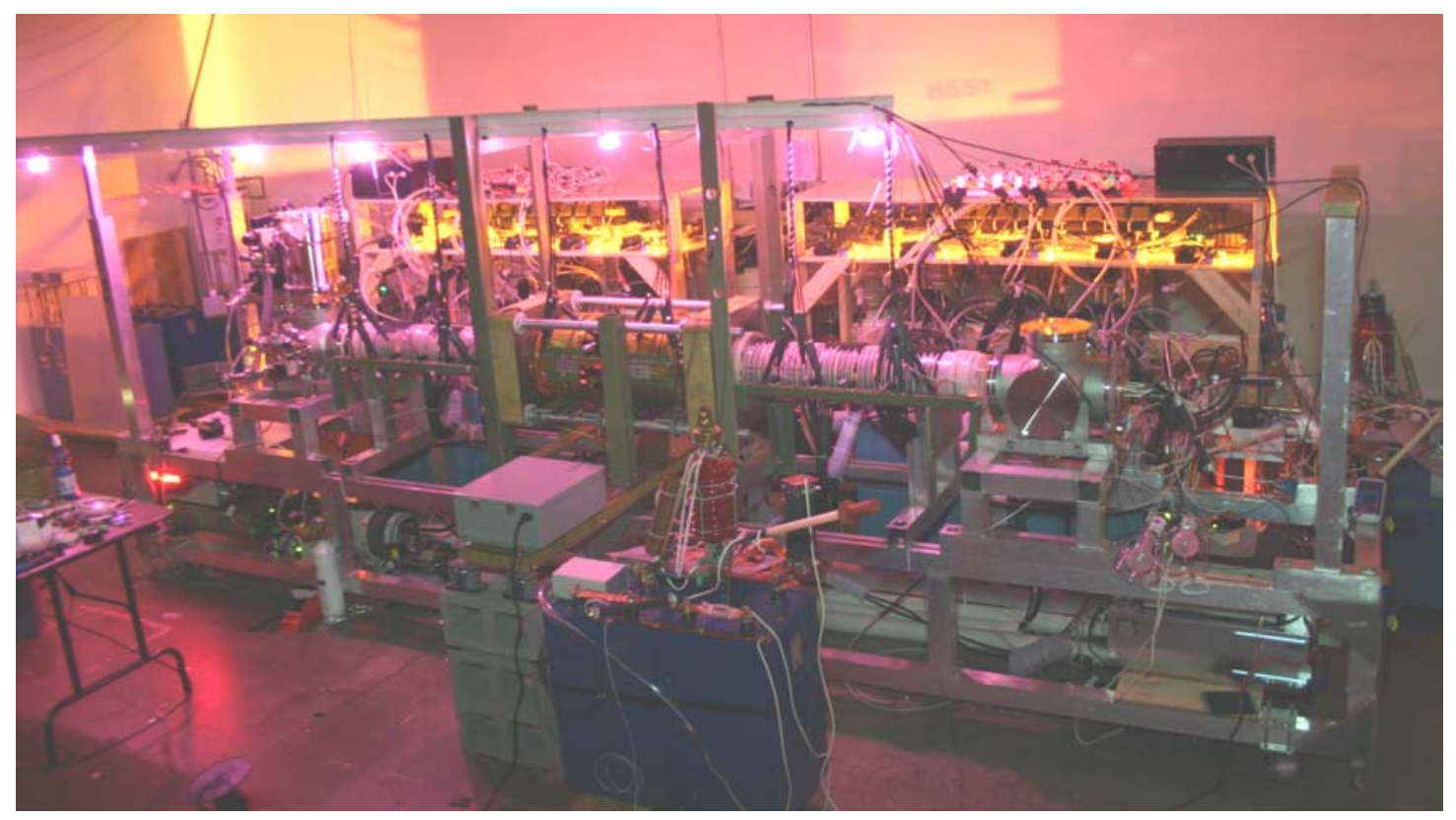

Figure 2. Photograph during operation of the IPA experiment at MSNW.

Recent results operating in the configuration shown in Figs. 1 and 2 were presented at the 2007 Innovative Confinement Concepts Workshop and published in the Journal of Fusion Energy. Details of the experimental setup can be found at the website. Several firsts were obtained in theses experiments. The principle results were:

(1) FRCs were simultaneously formed and accelerated to high velocity

The accelerator length was only $75 \mathrm{~cm}$ on each end. A terminal velocity of 250 $\mathrm{km} / \mathrm{sec}$ was attained inferring an average acceleration greater than $4 \times 10^{10} \mathrm{~m} / \mathrm{s}^{2}$. 
Both are records for FRC formation/acceleration. The ratio of FRC directed velocity to thermal velocity was greater than three. The FRC motion was thus highly supersonic (Mach 3).

(2) Merging and conversion of kinetic energy takes place on Alfven timescale

The rapid deceleration of the FRC is brought about by collision with the opposing FRC. From (1) the vast bulk of the energy was in the form of FRC kinetic energy. This kinetic energy was converted into thermal energy on the microsecond timescale at collision.

(3) Resulting FRC was quiescent and stable for $t>>\tau_{A}$.

Probably the most remarkable result of these experiments was the merging of the two hypervelocity FRCs into a stationary quiescent plasma that decayed on a timescale of $20-40$ axial Alfven times. This is the only merging experiment, including the several prior attempts with spheromaks, to ever achieve a stable, quiescent equilibrium. The FRC lifetime was several times that needed for the liner compression required for MTF.

(4) The merged FRC plasma temperature $400-500 \mathrm{eV}$.

From the MHD modeling of the experimental results, it is believed that the ions receive essentially all the converted translational energy. The ion temperature was on the order or greater than $400 \mathrm{eV}$.

(5) No rotational instability was observed during the quiescent decay of the FRC.

The best argument recently proffered for explaining such stability was the presence of a fairly large toroidal field component ( $>20 \%$ of the external axial field). The toroidal field can be generated by the simultaneous formation and acceleration of the FRC, and as such, it is likely that a toroidal field is generated in the IPA. The merging however would however tend to annihilate such a field as the induced poloidal currents that produce toroidal field are of opposite sign for the two merging FRCs. The rotational instability can be a serious limiting mode for the liner compression as the spin up parameter increases with compression to a smaller radius. The IPA experiment as it is currently configured will be able to examine whether the merged FRC continues to remain stable to this mode under compression.

These results warrant the approach proposed here as they address several difficulties that are inherent in the liner concept.

\section{D MHD Modeling of the Experimental Results}

The interpretation of the results of the initial operation of the IPA experiment was greatly aided by the use of the $2 \mathrm{D}$ resistive MHD code referred to as Moqui. By initializing the code with the experimental magnetic waveforms achieved in the experiments, the dynamic behavior of the FRC formation, acceleration and merging was matched by adjusting the initial flux and plasma inventory. The results of the calculations are shown in Fig. 3. 


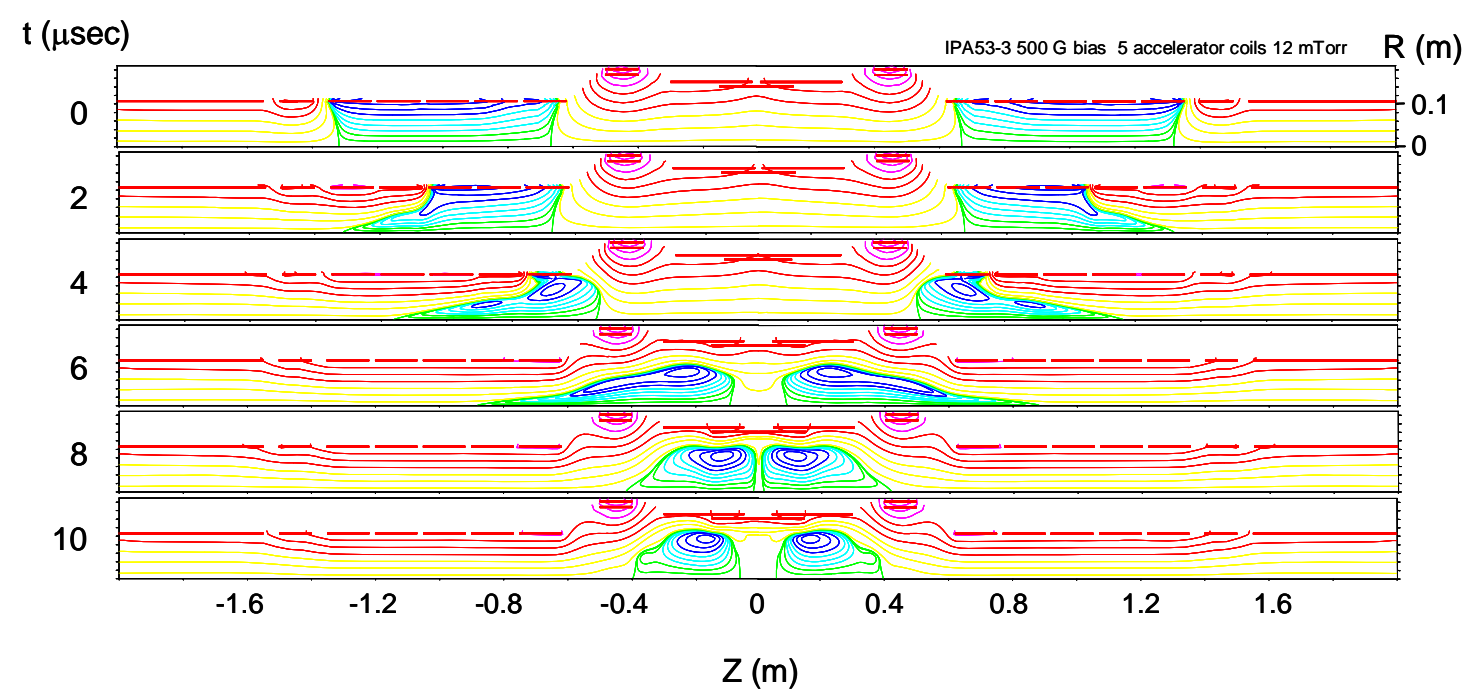

Figure 3. 2D MHD calculation of the IPA merging experiments.

The diagnostic comparison with the code results matched well at all times up to the post merging phase ( $\mathrm{t}=10 \mu \mathrm{sec}$ in Fig. 3).With the particular magnet geometry that was used with these initial experiments, the FRCs were predicted by the code to bounce off each other (albeit with virtually all the kinetic energy still being converted into thermal energy). This separation was not observed in the experiments. The magnetic data in the experiments indicated complete merging. The diamagnetic (excluded flux signals near the midplane of the IPA experiment illustrate this. In Fig. 4 the excluded flux signal at the center is plotted for the simultaneous merging and the case where only one IPA was operated. One can see that the rapid movement of the FRC through the region results in a rather small and transient signal. The small magnitude indicates the relatively low thermal component of the FRC for this case. Since the excluded flux is a direct indication of the line thermal energy of the FRC, the dramatic increase in excluded flux for the merging case is expected from the rapid conversion of translational energy into thermal for this case. The steady nature of the signal in time indicates that a stable equilibrium is

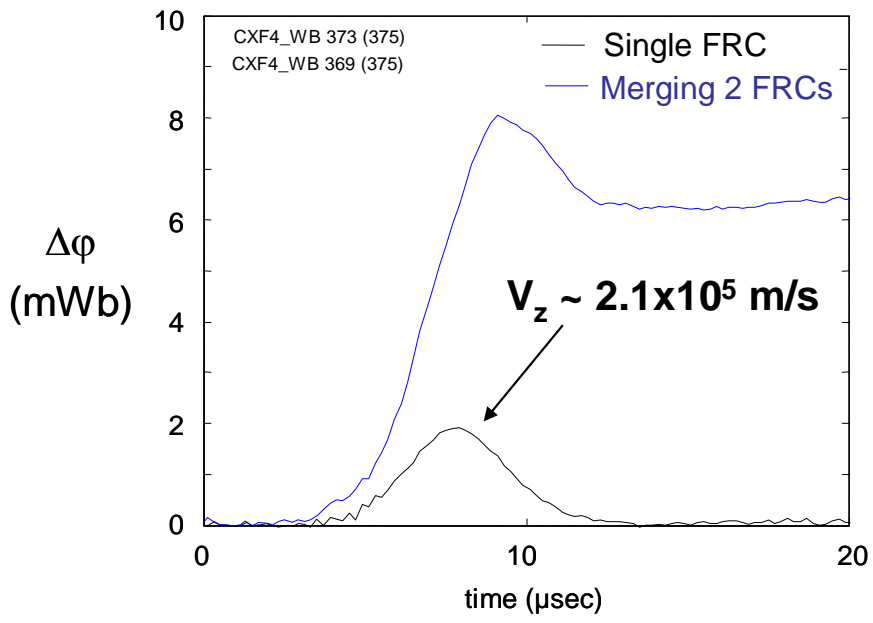

Figure 4. Centerplane excluded flux signals for merging and transiting FRC. established with little delay. It also indicates that full merging has most likely occurred. 
The rapid fall in FRC velocity and rise in ion temperature are predicted by the MHD calculations as indicated in Fig. 5. These results are fully consistent with the magnetic pressure balance temperature inferred by the midplane HeNe interferometer measurement of line density. The hot ion component is supported by the signal on the neutron detector shown in Fig. 7.

The other remarkable feature of the merging of two supersonic FRCs is the resulting radial size of the resulting FRC. All previous formation and translation results with FRCs formed in conventional reversed field theta pinches have resulted in FRCs with an equilibrium ratio of excluded flux radius to coil radius of 0.55 or less, i.e.

$$
\mathrm{x}_{\mathrm{s}} \cong \frac{\mathrm{r}_{\Delta \phi}}{\mathrm{r}_{\mathrm{c}}} \leq 0.6
$$
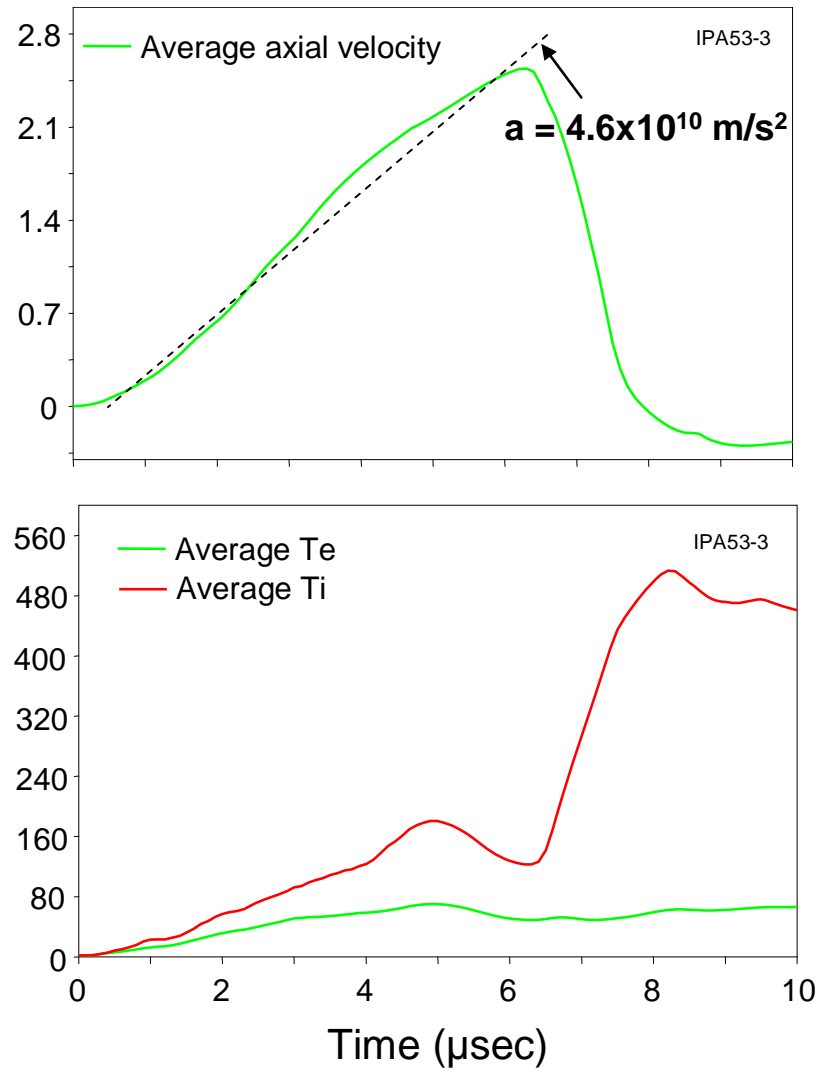

Figure 5. FRC velocity and temperature during

The separatrix radius is essentially the excluded flux the formation, acceleration, and merging of two FRCs. radius for negligible open field line plasma pressure as in the FRC. The merged FRCs form a resulting FRC with a much larger $\mathrm{x}_{\mathrm{s}}$ closer to 0.8 as indicated in Fig. 6. This might indicate a very much enhanced internal flux induced by the merger. This is the largest $x_{s}$ achieved for a hot $(>20 \mathrm{eV})$ FRC. This would be good news for liner compression phase as it provides for the maximum liner compression efficiency.

Such an amplification of the poloidal flux has been routinely observed in the formation of spheromaks, and could very well be happening here as well. It is not essential though as the flux inferred from the $2 \mathrm{D}$ calculations indicate enough flux to satisfy the requirements for the liner compression for $Q=1$ stated in Table $I$. Amplification of the flux by conversion of toroidal to poloidal flux would of course not be seen in a 2D calculation since according to Cowling's theorem there must an axis symmetry breaking perturbation to allow for such a transformation. 


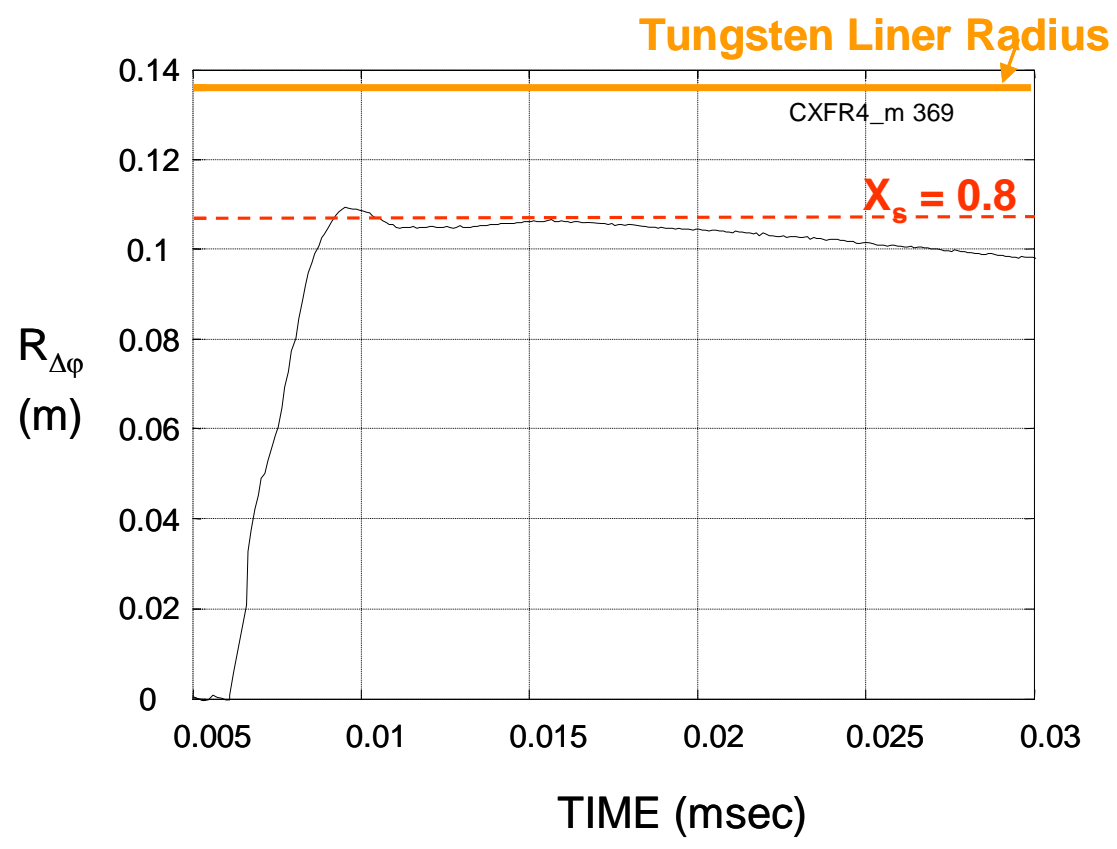

Figure 6. Excluded flux radius of merged FRC. Quartz tube was lined with a 50 $\mu \mathrm{m}$ tungsten foil liner that formed the flux conserver during the first $25 \mu$ sec after the merger.

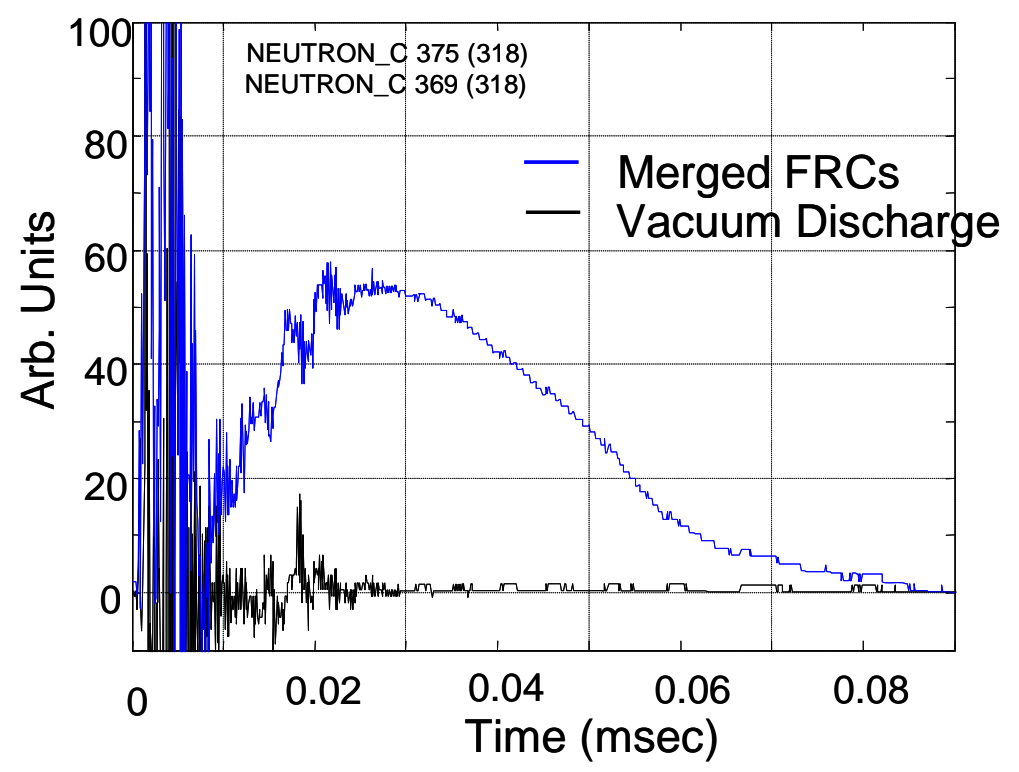

Figure 7. Neutron detector signal for vacuum and plasma merging. The neutron detection was performed by a photomultiplier tube monitoring emission from a scintillator encased in a lead and aluminum shield positioned at the midplane of the experiment. 


\section{The new IPA-C Experiment}

With the IPA concept, the energy needed to achieve fusion conditions is transferred to the FRC plasmoid via simple, relatively low field acceleration/compression coils, and it is believed that this process can be made very efficient and avoid the need for inefficient methods such as neutral beams to reach fusion temperatures. We believe that a validation of the concept can be performed at a fraction of the cost and time now being spent on the more developed fusion systems (e.g. the ITER project @ \$10 B, and 20 years). This is possible due to the unique nature of the plasma source that is to be employed and the method by which it is brought to fusion conditions.

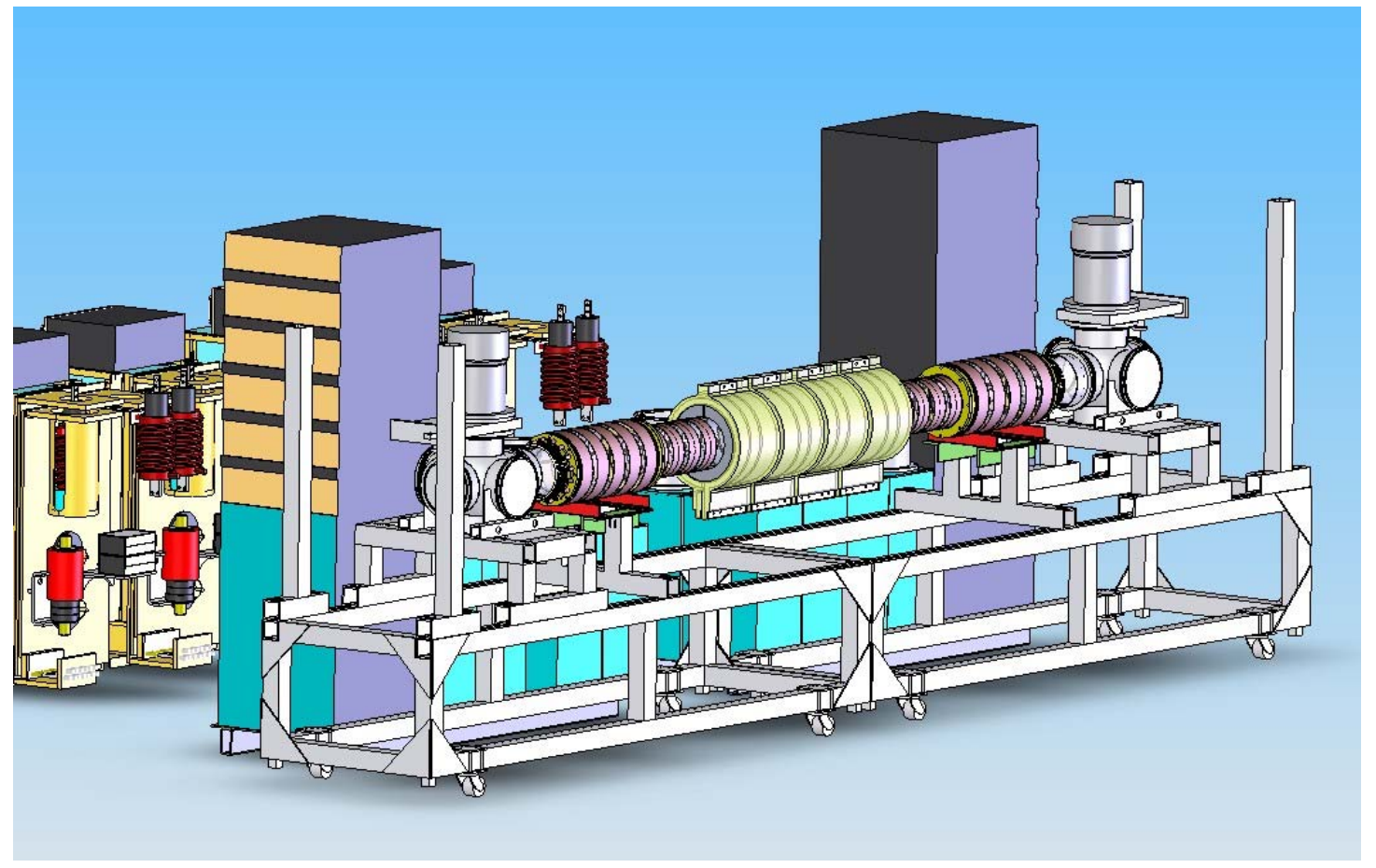

Figure 8. CAD rendering of the IPA-C experiment at MSNW. FRCs are to be simultaneously formed and accelerated to $400 \mathrm{~km} / \mathrm{sec}$ over a $\sim 1.5$ meter length. The two FRC's are then merged, rapidly thermalizing the motional energy forming a hot, stationary, long-lived plasmoid. This plasmoid is further compressed by flux compression employing a set of four 3-turn coils in the merging region. IPA-C is an upgrade of the initial experiments that successfully demonstrated plasmoid acceleration and merging. Improvements include a larger source with staged compression-acceleration. The experiment has been designed to access the reach temperature range with significant D-T fusion cross-section (2-3 keV).

In addition to IPA, there is an experiment underway, the PHD experiment at the University of Washington, that is also evaluating the magneto-kinetic compression of the FRC plasmoid to fusion breakeven $\mathrm{Q}_{\text {fus }}=1$ conditions. In this approach, the energy required to bring the plasma to fusion conditions is transferred to the FRC by 
magnetically accelerating the plasmoid to high velocity as well. The conversion of this kinetic energy into thermal energy is accomplished in a different manner. Here it will be slowed by compression into a tapered coil that merges into a high field burn chamber. Alternatively, a pair of FRC plasmoids can be accelerated and self compressed axially by collision in addition to being compressed radially via an axial magnetic field, solid liner, or plasma liner. This second approach is the one being pursued at the MSNW laboratory. The next set of experiments will be directed toward compressing this merged FRC plasmoid to fusion temperatures. This new IPA-C device will serve as the test bed for the Proof Of Principle (POP) experiments validating this approach to fusion. A schematic of the experiment is shown in Fig. 8.

\section{Heating and compression of FRC plasmoids}

The radial compression inward and the deceleration of the plasmoids are performed in staged manner to provide for a very rapid evolution of the FRC into a state fusion burn. The timescale for forming and accelerating the FRC $\left(\tau_{\mathrm{acc}}>>1 \mu \mathrm{s}\right)$ can be much longer than the time that the energy is thermalized in the merging ( $\tau_{\text {heat }}<1 \mu \mathrm{s}$ ). The consequence is a greatly reduced demand on the power delivery systems as the energy can be accumulated over a time interval of several microseconds far from the merging/compression region. The FRC can be easily translated over several meters so that a large stand-off distance can be maintained between the reactor and driver. Critical to the practical implementation of fusion is the unique ability of the FRC plasmoid to be translated far from the plasma production and energy delivery systems, allowing these systems to be properly shielded from the burn chamber and blanket.

Both IPA-C at MSNW and the Pulsed High Density (PHD) fusion experiment at the UW can be employed to achieve fusion conditions in this manner. As mentioned, the main difference between the approaches is the way in which the energy is thermalized to bring the plasma to fusion temperatures. For the POP, the IPAC approach is simpler in that the transfer of the energy is automatically achieved as the net axial momentum is zero at all times, and the process does not require the action of any external coil or structure to decelerate the FRC. Since it is not required to attain a fusion gain greater than unity for the POP, the advantage of the much larger plasma source and burn chamber in the PHD approach is not needed. For the POP demonstration the simplicity, low cost, and compactness of the IPA-C as a test bed is clearly preferable. Given the strong commonality of both the technology and physics between both devices, the advance of one approach will contribute to the advancement of the other.

In IPA-C the energy that is required for the compression and heating of the FRC plasmoid is derived from both the magnetic compressional energy of the central magnets and the axial kinetic energy of the FRC's motion prior to compression. The acceleration of the FRC and the final compression can be well separated. There are thus two key parameters that naturally emerge to describe the compressed FRC plasmoid, one being the final external confining axial magnetic field, $\mathrm{B}$, and the other being the FRC plasmoid energy $E_{p}$. 


\section{FRC equilibrium, confinement and fusion yield scaling for IPA-C}

The FRC is a prolate, compact toroidal plasma that is magnetically isolated from the axial magnetic field used to confine it. The closed magnetic field structure is the result of currents induced during formation and acceleration of the FRC. Thus, in a sense, it is "self confined" forming what is called a plasmoid. It has topologically the simplest closed field line structure and the highest equilibrium $\beta$ (the ratio of plasma to magnetic energy density) of any magnetically confined plasma. The prolate FRC equilibrium is characterized by the following relations:

$$
\begin{aligned}
& \text { Radial Pr essure Balance: } P_{0}=n_{0} k T=\frac{B_{\text {ext }}^{2}}{2 \mu_{0}} \\
& \text { Axial Pr essure Balance: }\langle\beta\rangle=\int_{0}^{r_{r}} \frac{2 \mu_{0} P(r)}{B(r)^{2}} d r=1-\frac{1}{2} x_{s}^{2}
\end{aligned}
$$

Although other FRC experiments have been aimed in the direction of achieving a relatively low density steady-state reactor embodiment, there were several reactor studies that took advantage of the translatability and compressibility of the FRC. A limitation for these studies was the lack of sufficient data regarding the scaling of FRC confinement. Since these studies, many experiments over a wide range of conditions have been performed, and considerable data has been accumulated from various FRC experiments that span over two orders of magnitude in density and an order of magnitude in radius. The scaling has been stated previously in terms of inferred quantities that are not directly measured $\left(\varphi_{\mathrm{p}}, \rho_{\mathrm{i}}\right.$, etc). For the purposes here, it is better to state confinement in terms of the basic external parameters that can be easily and accurately measured from all experiments. The observed particle confinement, cast in these terms, yields the following scaling:

$$
\tau_{\mathrm{N}}=3.2 \times 10^{-15} \varepsilon^{0.5} \mathrm{x}_{\mathrm{s}}^{0.8} \mathrm{r}_{\mathrm{s}}^{2.1} \mathrm{n}^{0.6},
$$

where $\mathrm{x}_{\mathrm{s}}$ is the ratio of the FRC separatrix radius, $\mathrm{r}_{\mathrm{s}}$ to coil radius, $\mathrm{r}_{\mathrm{c}}$, and $\varepsilon$ is the FRC separatrix elongation, $l_{s} / 2 r_{s}$. It was found that the only significant energy loss channel for the FRC was particle loss, so that the particle confinement scaling of Eq. (3) will be assumed for the energy confinement scaling in determining reactor conditions. From this empirical scaling, came the realization that the improved confinement with density more than compensates for the size scaling as one reduces the size of the FRC. It is noteworthy that the confinement scaling observed for tokamaks has a very similar dependence on size and density. The need for a very large toroidal field and complex tokamak coil geometry however limit the amount of field increase and therefore size reduction possible for the tokamak.

We believe that the first and possible best application of the IPA is as a fusion neutron generator. IPA-C could be used in this context for applications ranging from 
neutron diagnostics to fusion-fission hybrids and waste transmutation. The methodology is obviously very similar to the application for electric power generation from only the fusion reaction. A crucial difference is that these applications can be successfully attained at much lower $\mathrm{Q}$ values. Following this path allows for fusion to make an immediate contribution to the global energy solution. Enabling alternate fission cycles based on thorium provide for the elimination of the long-lived radioactive waste. This capability, together with the ability to produce essentially unlimited quantities of additional fuel, obviate the need for a pure fusion based power source for thousands of years.

The calculation for the neutron yield is obviously similar to that for D-T fusion gain. However, since the metric is neutrons, there is no need to consider whether there is sufficient energy production to supply enough thermal energy for conversion into electrical energy for sustainment (i.e. breakeven). Using fusion neutrons to catalyze the fission process acts as an energy amplifier. The breakeven condition criterion then becomes of secondary consequence. The optimization therefore differs somewhat from the usual Lawson argument. It basically comes down to what conditions produce the maximum neutron flux for a given input energy to the plasma. It is the total plasma input energy that is the controlling variable, and it applies to any device where the plasma energy must be supplied from outside sources. Virtually all the costs in neutron production will scale with this energy. This same assessment applies to all current and future fusion experiments since it requires close to fusion ignition conditions before input power is no longer important, as it is for fission. There could be other significant issues such as the need for current drive, large orbit ion beams, etc. Fortunately these are not required for the pulsed FRC plasmoid fusion considered here. Here the energy input is the only parameter of importance.

The neutron production rate $\mathrm{dN}_{\text {neu }} / \mathrm{dt}(\mathrm{n} / \mathrm{sec}$ ) for D-T fusion systems is given by

$$
\frac{\mathrm{dN}_{\text {neu }}}{\mathrm{dt}}=\int_{\text {vol }} \mathrm{n}_{\mathrm{D}} \mathrm{n}_{\mathrm{T}}\langle\sigma \mathrm{v}\rangle \mathrm{dVol}
$$

where $n_{\alpha}$ is the number density of species $\alpha$, and $\langle\sigma v\rangle$ is the reaction rate parameter for the fusion reaction of interest (in this case D-T) and is in units of $\mathrm{m}^{3} / \mathrm{s}$. For pulsed systems such as the FRC plasmoid the relevant quantity is the total neutron yield per pulse. Equation (4) should then be integrated over the lifetime of the plasmoid. For the purposes here it will be sufficient to multiply the left hand side of Eq. (4) by the confinement time expressed in Eq. (3). During the decay of the FRC the plasma density and temperature are maintained as the product must be in radial pressure balance with the external magnetic field (see Eq. (2)). It will be assumed that $n_{D}=n_{T}=1 / 2 n$ where $n$ is the total ion density. Equation (4) can be simplified for the FRC to:

$$
\mathrm{N}_{\mathrm{neu}} \cong \frac{1}{4} \mathrm{n}^{2} \cdot \mathrm{Vol} \cdot \tau_{\mathrm{N}} \cdot\langle\sigma \mathrm{v}\rangle
$$

The parameters that can be directly influenced in the IPA-C device will be the plasma energy and the compression magnetic field. The plasma energy, $\mathrm{E}_{\mathrm{p}}$ is introduced through 
the acceleration of the FRC plasmoid and created by the thermal conversion of the FRC plasmoid directed energy as well as compression by the external magnetic field. This energy is imparted almost exclusively to the plasma ions $\left(T_{i}>>T_{e}\right)$. The final plasma energy can thus be stated as:

$$
\mathrm{E}_{\mathrm{p}} \sim\left(\frac{3}{2} \mathrm{nkT}_{\mathrm{i}}\right) \cdot \mathrm{Vol}
$$

From Eqs. (1) and (2), the plasma energy can be restated as:

$$
E_{p}=\frac{B^{2}}{2 \mu_{0}} \pi r_{s}^{2} l_{s}
$$

We wish to state the neutron yield (Eq. (5)) as a function of the controllable variables, i.e. the plasma energy and magnetic field. Solving for $n$ in Eq. (1), and with Eq. (7), Eq. (5) can be restated using the scaling expressed in Eq. (3) as:

$$
\mathrm{N}_{\text {neut }}=2.42 \times 10^{31} \mathrm{~B}^{2.2} \mathrm{E}_{\mathrm{p}}^{3 / 2} \mathrm{r}_{\mathrm{c}}^{0.6} \mathrm{x}_{\mathrm{s}}^{1.4} \frac{\langle\sigma \mathrm{v}\rangle_{\mathrm{DT}}}{\mathrm{T}_{\mathrm{i}}^{2.6}}
$$

The ratio of the FRC plasma radius to coil radius, $x_{s}$, is a function of the initial closed poloidal flux produced during FRC formation. This flux can be stated in terms of this ratio and the external field and coil radius:

$$
\varphi_{\mathrm{p}}=\mathrm{x}_{\mathrm{s}}^{3} \mathrm{r}_{\mathrm{c}}^{2} \mathrm{~B}
$$

The neutron yield can now be stated solely in terms of parameters that are experimentally determined:

$$
\mathrm{N}_{\text {neut }} \cong 2.6 \times 10^{31} \frac{\mathrm{B}^{7 / 4} \mathrm{E}_{\mathrm{p}}^{3 / 2} \sqrt{\varphi_{\mathrm{p}}}}{\mathrm{r}_{\mathrm{c}}^{1 / 3}} \frac{\langle\sigma \mathrm{V}\rangle_{\mathrm{DT}}}{\mathrm{T}_{\mathrm{i}}^{2.6}}
$$

where the ion temperature is measured in $\mathrm{keV}$, and the temperature dependent terms have been grouped together. This second temperature dependent term is interesting in that there is a strong temperature weighting to the usual fusion reaction rate toward lower temperatures. This stems from the fact that if one ignores the temperature dependence on cross section, for a given plasma energy and compression field, it is more favorable to increase density at the expense of temperature (see Eq. (5)). Of course this cross section is highly dependent on ion temperature.

The effect of the weighting is significant as can be seen in Fig. 9. The $\mathrm{T}^{-2.6}$ weighting moves the optimum plasma ion temperature to a much lower value. In fact, this leads to a temperature somewhat lower than that found for an optimization like the Lawson 
criterion which is constrained only by radiation losses. This reflects the favorable density scaling observed in past FRC experiments (see Eq. (3)). An expanded view is found in Fig. 10. From this figure it can be seen that there is little advantage to be had in producing an ion temperature much greater than 5 to $6 \mathrm{keV}$.

The strong scaling of neutron production with both the plasma energy and magnetic field make a fusion driven system based on the FRC plasmoid far more advantageous than other approaches to neutron generation such as the accelerator driven systems (ADS) based on neutron spallation. Here the neutron yield scales only with the energy of the proton beam at best. The proton energy employed in spallation sources is in the $\mathrm{GeV}$ range and is many orders of magnitude larger than the optimum $8 \mathrm{keV}$ plasma ion temperatures of the FRC

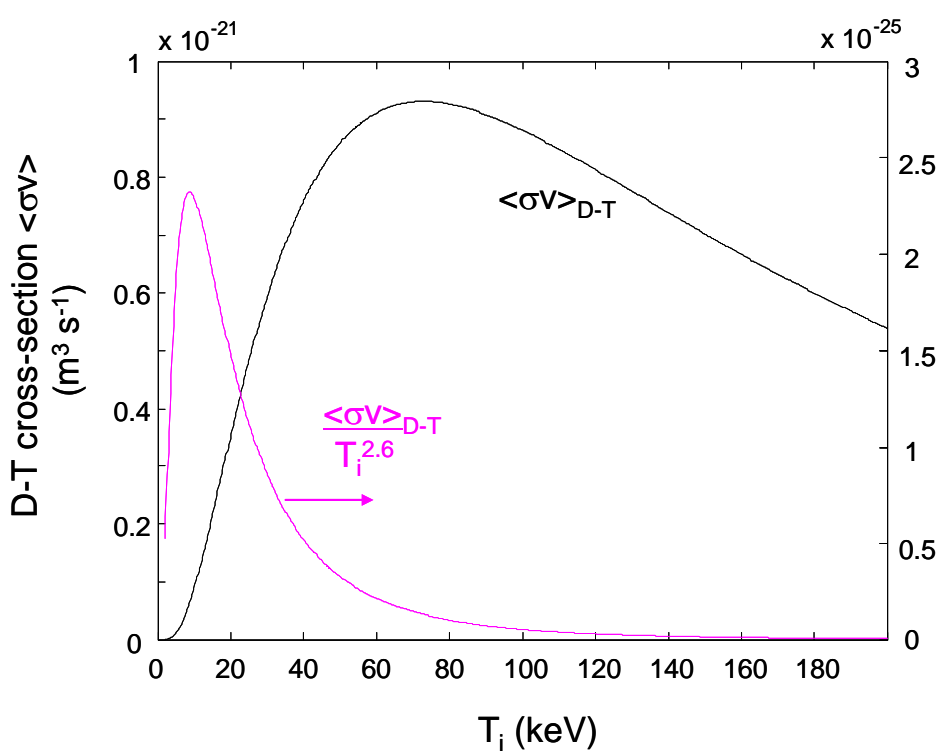

Figure 9. Integrated $D$-T cross-section for Maxwellian ion distribution as a function of ion temperature. Magenta trace reflects $1 / T^{2.6}$ multiplier (see Eq. 10).

plasmoid.

Particle accelerators of kilometer scale are required for ADS systems rather than meters envisioned here. Yet even with this disadvantage, accelerator systems have been shown to be cost effective for the generation of nuclear power. A fusion driven system (FDS) should be even more promising.

The intent of the modifications to IPA-C is to provide both a model for the MTF approach, but to also establish the approach as a valid method for the generation of fusion neutrons. Satisfying the first goal - a suitable target plasma for compression - would

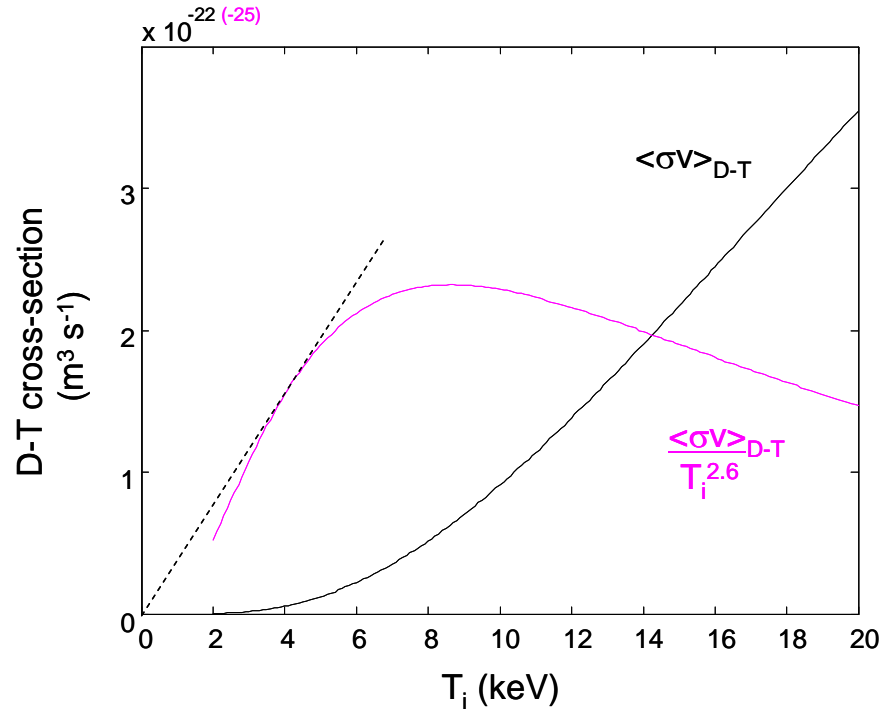

Figure 10. Expanded low temperature region of Fig. 9. 
assure that the physics and technical approach is sound for the second. The new IPA-C facility will actually comprise the essential Proof of Concept test bed. The ultimate challenge will be the demonstration of a large neutron yield that requires that all the dependences found in the previous FRC confinement scaling (Eq. (10)) be correct and accurate predictors. By having a target neutron yield far greater than that currently attainable, a sufficiently larger yield would put the scaling on solid footing. It would also allow for a better determination of the parameters required for a yield sufficient for nuclear energy generation by a FDS. Even if the yield was not as favorable as implied by Eq. (10), there is enough margin in the scaling to still achieve the necessary production with only modest increases in energy input or rep rate. After all, it is just as likely that the results could be much better making the next step easier.

\section{Design, Construction and Operation of IPA-C}

The first objective was building an experimental platform for the successful test of both goals. Confidence that the properly designed experiment can achieve the desired results is supported by comprehensive resistive 2D Magnetohydrodynamic (MHD) calculations on the one hand, and extensive experience with power generation equipment of the same type as will be employed in the IPA-C experiment. The latter is reflected in the IPA device at MSNW and the new modifications. The modifications required for the MTF and FDS goals can be seen in comparing Fig. 1 and Fig. 8. A discussion of the modifications to the previous IPA experiment is in order so that the motivation behind these modifications can be more clearly understood.

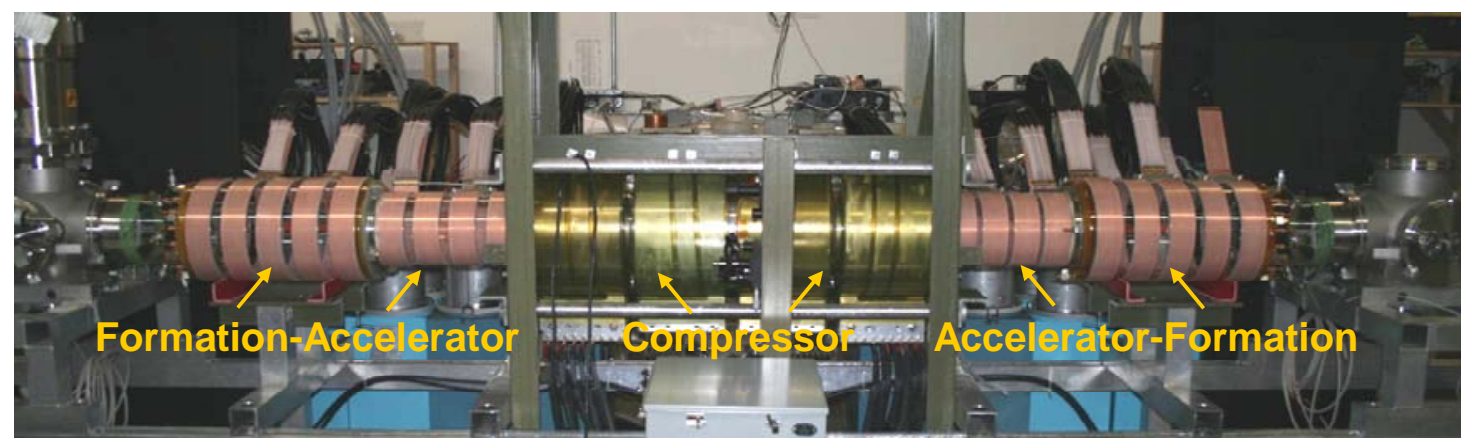

Figure 11. IPA-C configuration after magnet assembly

It is clear from Eq. 10 that one desires the highest energy FRC compressed to the largest field consistent with available power sources. The plasma energy is leveraged by the compression field so that the compression field is critical. From Eq. (10) it is clear that the scaling with size and flux is not nearly as strong as field and plasma energy, so that there is little advantage in trying to maximize FRC poloidal flux or compressing into a small coil. There are hidden dependences here however. A larger starting plasma will have more energy and mass. This will lead to a much larger kinetic energy and thus final energy. A larger plasma source was thus included in the new IPA-C configuration. It also takes less energy to provide for a given compression field in a smaller coil. The 
compression coil radius was thus reduced from $15 \mathrm{~cm}$ to $10.5 \mathrm{~cm}$. A greater degree of radius reduction was not practical for several reasons - bank energy coupling and vacuum system issues to name a few. The new IPA-C system was designed, and the initial assemble has been completed. A picture of the new configuration is shown in Fig. 11.

As can be seen in the figure, the new dynamic formation section is in addition to the accelerator section from the old IPA setup (see Fig. 1). This required eight additional coil and capacitor modules. These were provided by MSNW from another project. In addition, the old accelerator modules were upgraded form a nominal $25 \mathrm{kV}$ charge to a full $40 \mathrm{kV}$. The entire formation/acceleration section is now much more capable in increasing the thermal energy of the target FRC. Special low inductance feeds and magnets were also fabricated to improve the energy coupling in the formation and accelerator sections. The additional plasma energy should be a factor of 5 to 6 larger than that achieved in the initial IPA experiments.

The other major improvement was an upgrade of the compression banks. We are limited to the three pair of power supply modules that were employed on the original IPA compression coils. These are legacy supplies from the old LSX experiment. The issues are age, stray inductance, and the use of ignitrons for switching. There is nothing that can be done about the age, but the other two aspects could be, and were considerably improved. The ignitron based switches limited the voltage to $\pm 15 \mathrm{kV}$ while the capacitors are rated for $25 \mathrm{kV}$. In addition there were several hundred nanoHenries of stray inductance associated with these

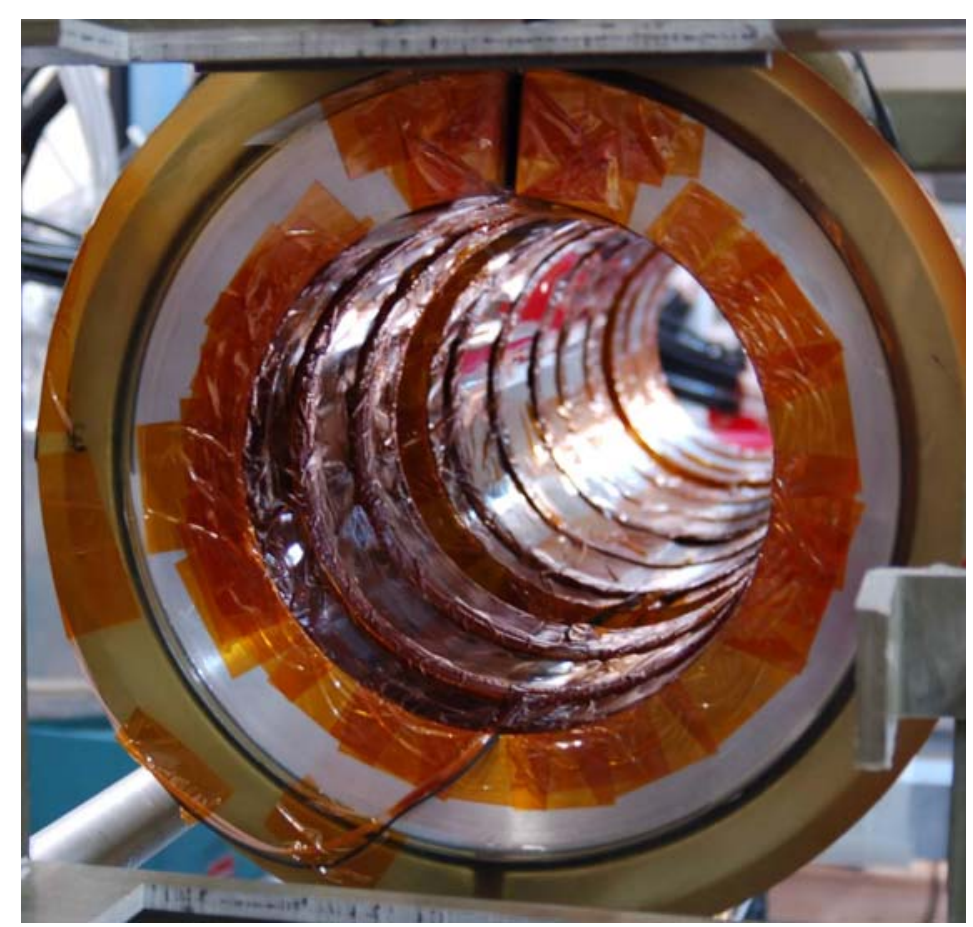

Figure 12. Aluminum flux concentrator installed in the original 3 turn compression magnets. An array of nine diamagnetic loop pairs are held in place by Kapton tape.

switches. All twelve ignitrons were replaced with a pseudo-spark thyratron switch on both the start and the crowbar. The inductance of these switches is $\sim 25 \mathrm{nH}$, and can easily be operated at $\pm 25 \mathrm{kV}$ providing the full $270 \mathrm{~kJ}$ of stored energy for compression. The compression coils are 3-turn solenoids to provide for better coupling to the old modules, so that the coupling is now quite good (85\%). The goal was to increase the maximum vacuum compression field from $0.8 \mathrm{~T}$ to $2 \mathrm{~T}$. This requires a factor of $\sim 6 \mathrm{x}$ increase in energy for the same coil configuration. The increase in voltage and coupling 
efficiency will provide a factor of 3x. The final increase must come from a reduction in the volume filled. Decreasing the length of the compressor coil section turns out not to be a viable option. With the large increase in $E_{p}$ from both the larger source and more powerful accelerator, a much more powerful compression will be required to maintain the FRC equilibrium length let alone decrease it. This is due to both the weak dependence on FRC length with compression which scales with only the 0.4 power of B. However the length increases linearly with $\mathrm{E}_{\mathrm{p}}$ for the same $\mathrm{B}$. A much larger compression field bank will be a key element of the next phase of development of IPA-C, but for now we can not significantly reduce the accelerator length if we wish to substantially increase the FRC energy as well. The coil radius is quite a different matter, and that is the way in which we can achieve a $2 \mathrm{~T}$ compression field with the same physical bank.

A smaller compression coil radius requires a new magnet. By reducing the coil size to that of the original IPA accelerator, a new vacuum tube of the same radius can be used for both the accelerator and the compression section making life much simpler. This favored a coil reduction to an inner radius of $10 \mathrm{~cm}$.

A re-machining and potting of the high voltage coils would be prohibitively expensive as well as adding a large delay from manufacture. A smaller magnet bore can be achieved by the insertion of what is referred to as a flux concentrator. It consists of a large conducting annulus that is split radially in at least one azimuthal location to allow for flux entry. Such a large bore Aluminum shell was obtained and machined to fit precisely between the existing three turn coil and the quartz vacuum chamber in the compression section. A picture of the coils with the shaper can be found in Fig. 12.

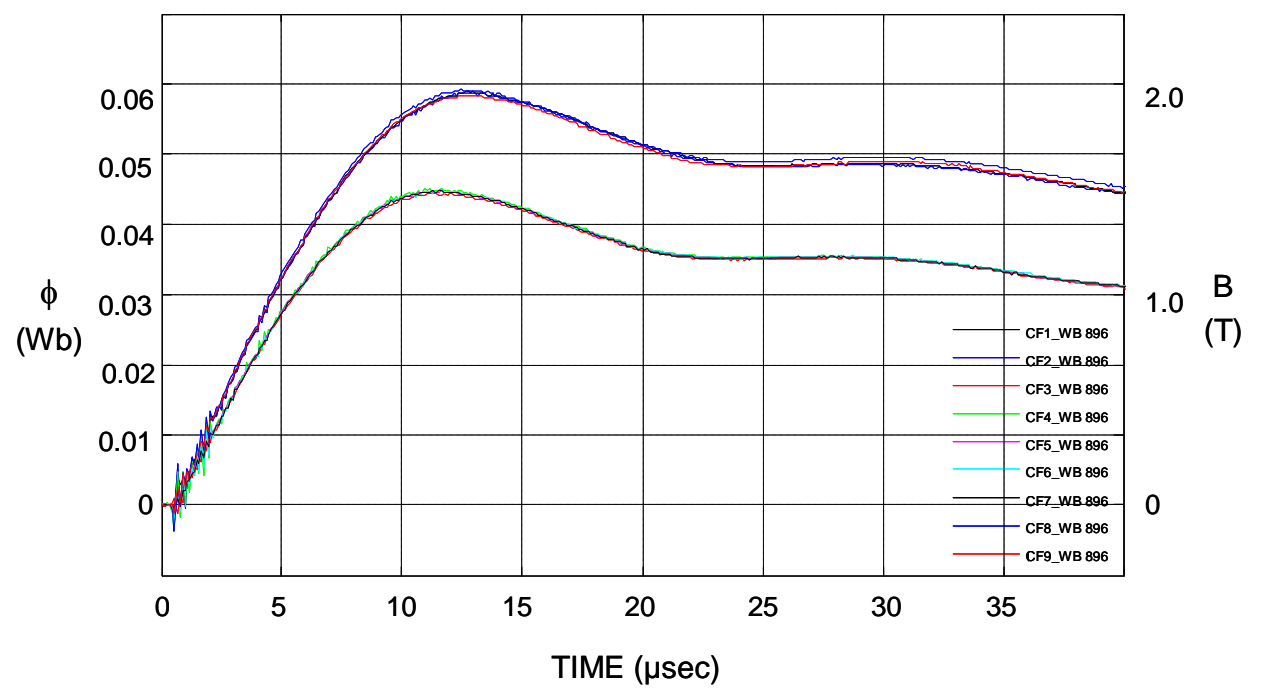

Figure 13. Time history of the axial vacuum magnetic flux in the IPA-C compression section. The traces are from the array of nine flux loops positioned axially along the four coils.

There is an additional advantage in employing a flux concentrator in that it also provides for a better flux shaper. It completely eliminates non-axially symmetric 
magnetic field errors from the axial current flow that occurs with the use of a multi-turn solenoid. The results form the initial testing of the compression modules can be found in Fig. 13. These results reflect operation at $20 \mathrm{kV}$ of a maximum $25 \mathrm{kV}$. Operation at full charge will await operation with plasma. In this figure the flux loop signals from all nine loops are recorded. The loops are positioned every $10 \mathrm{~cm}$ so that an accurate measure of FRC energy and confinement can be made. The desire was to achieve a mirror configuration that will add axial as well as radial compression to the FRC plasmoid. The use of a relatively strong mirror will also center and dampen axial oscillations observed after merging. To achieve this, only one module pair is employed for the two center coils which are mounted onto a single flux concentrator. A separate flux concentrator is employed in each end coil. Each end coil is then powered by a full module pair. The resulting flux signals indicate good symmetry as well as the excellent flux maintenance inside the individual flux shells.

The presence of the plasma will increase the magnetic field of the compressor by an amount equal to the flux displaced. For a final separatrix to coil radius of 0.5 anticipated for the IPA-C experiments, this will increase the vacuum field by a factor of $1 /(1-0.25)=$ 1.33 .

\section{New Preionization System}

Key to achieving good azimuthal symmetry as well as minimizing upstream and compression chamber neutral density is a good preionization system. The ideal preionization would locate the entire requisite plasma inventory near the vacuum tube wall just prior to field reversal. This would minimize flux loss through reversal as well as position the plasma radially near the magnetic null - the ultimate destination for most of the plasma in equilibrium. Full ionization would also minimize the energy lost from the initial compression and acceleration in bringing the plasma to the fully ionized state. With an energy loss on the order of $50 \mathrm{eV}$ per ionization and dissociation of a Deuterium
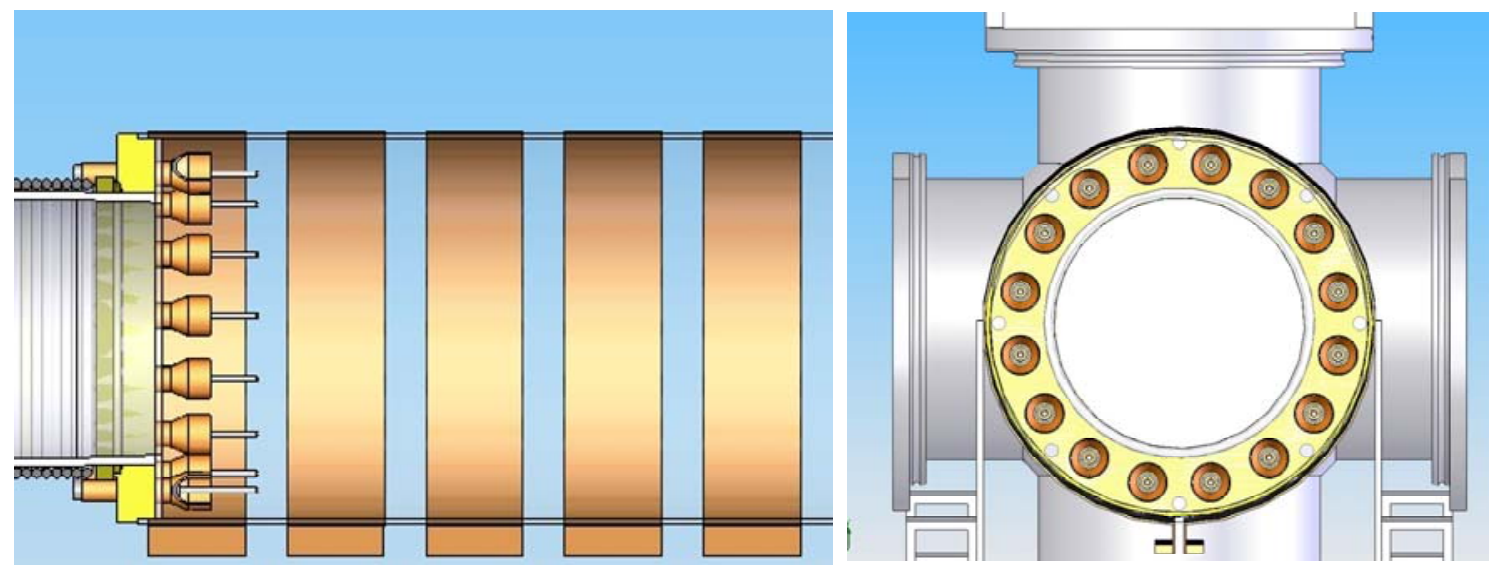

Figure 14. CAD rendering of the 16 coaxial discharge sources in each formation section on IPA-C. 
ion, this is a significant component that could go instead into heating and accelerating the plasma. Full ionization also inhibits instability as the energy is immediately available to heat the ions providing FLR stability to the flute modes commonly seen to grow in the initial phase of the non-equilibrium FRC.

The preionization system was designed to provide as close to this ideal case as possible. In order to produce the dense plasma annulus near the vacuum wall, an array of coaxial plasma guns have been constructed and positioned at the upstream end of the formation region. A CAD schematic of the system can be found in Fig. 14. As can be seen, these plasma sources are positioned as close to the wall as practical. They are located

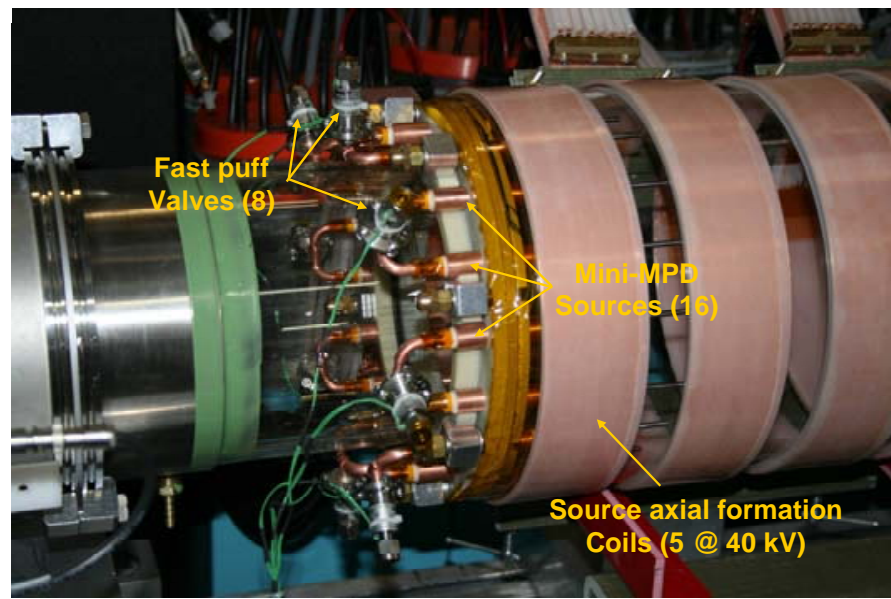

Figure 15. Preionization system on IPA-C. under the first reversal coil so that the plasma is deposited onto the reverse bias field and allowed to flow axially along the axial bias field to the other end of the formation section. There are sixteen sources on each end of the IPA-C device.

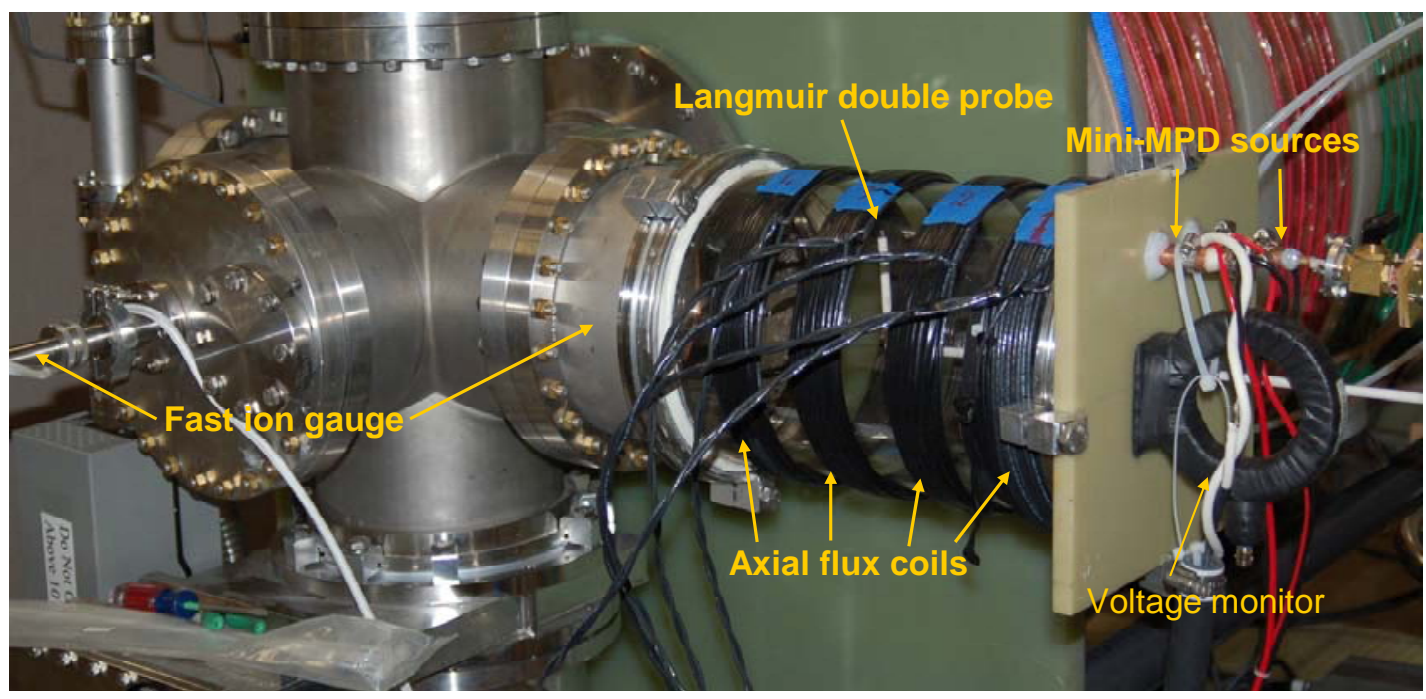

Figure 16. Setup for evaluation of the mini-MPD plasma sources.

A good deal of effort was put into the design and testing of these sources. This was aided considerably by support from the phase I work on the plasma liner compression experiment. For this application a dense plasma sheath is required to form the plasma 
liner. For the liner application, all 32 (and likely more) guns will be placed at both ends of the $14 \mathrm{~cm}$ radius quartz chamber. Sixteen coaxial sources were deemed a sufficient test of this approach, and fit nicely into the role required here for startup. For the high mass liner each source will be fed by a fast puff valve. The mass flow from these valves can be made quite high as they can be operated at up to 50 atmospheres. The mass flow inferred from tank tests is on the order of several grams per second. To achieve a $1 \mathrm{mg}$ sheath mass in $20 \mu$ sec (target values for the PLC experiments) at least 25 valves will be required. For the $0.1 \mathrm{mg}$ final FRC mass desired here far fewer are required. To make a good test of the technique a compromise was reached. Each end employs 8 puff valves with each feeding two sources. A picture of the installation on one end is shown in Fig. 15.

The basic behavior of the plasma sources was studied with the use of several diagnostics on a separate vacuum chamber. This chamber had both a capacitive manometer for accurate determination of the total mass flow, and a fast ion gauge that was employed for obtaining the time of arrival of the neutral gas downstream of the plasma guns. The setup is illustrated in Fig. 16.

The plasma source was based on a somewhat smaller version of the arcjet Magneto-plasmadynamic

(MPD) thruster. These devices show very low erosion and stable operation at near the full ionization desired here. They also operate at mass flows in the range desired $(0.2-2 \mathrm{~g} / \mathrm{sec})$. The key is to operate at a high enough current so that the flow is dominated by the $\mathrm{JxB}$ force on the plasma, but not too high where the neutral flow into the thruster is less than the plasma flow out. Violating this condition is usually correlated with enhanced sputtering and electrode erosion, and is referred to as "onset". The presence of an axial field is actually found to help suppress this. Since we are primarily interested in ionization and not flow, it was fairly easy to operate below onset threshold and still achieve nearly full ionization.

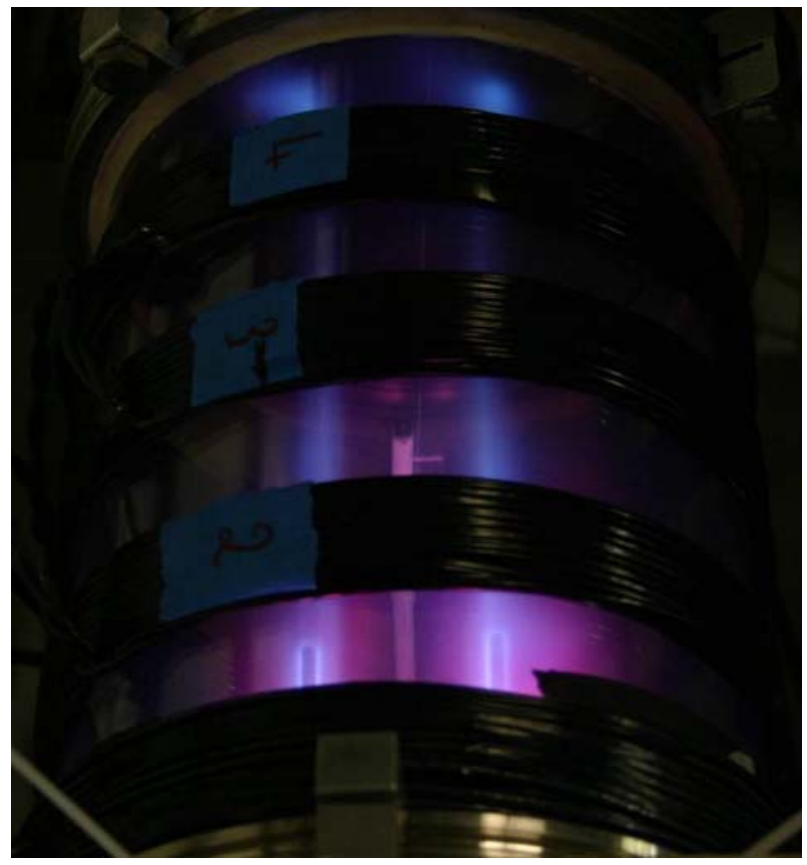

Figure 17. Time integrated image of discharge 2608 taken from above discharge tube (see Fig. 16).

There was one important effect from the method of operation here that is not found in normal operation of an MPD thruster. The bias magnetic field is introduced on a timescale that is too short to allow for the axial field to penetrate the copper anode keeping the axial field outside of the thruster. This exclusion also leads to a strong magnetic cusp at the thruster exit as the axial field 
returns downstream of the anode. The plasma must overcome this magnetic "hill" to leave the thruster. It was found that this cusp field effectively bottled up the plasma flow unless the cathode extended well beyond the anode into this higher field region. The path of the discharge could then follow the natural magnetic contours, and the plasma escapes. The discharge extension beyond the thruster body downstream is easily observed in the digital images as can be seen in Fig. 17.

It is also clear from this picture that the plasma is well magnetized. The emission is somewhat deceptive here. The light is produced by the electron excitation of the few downstream neutrals which are concentrated radially near the tungsten cathode. The double Langmuir probe was positioned at the radius of the plasma sources and could be located at arbitrary azimuth. With the probe positioned between the plasma guns as seen in Fig. 17, the ion flux is roughly half that directly downstream of a gun. Initially plasma stream is narrow but by the peak signal at $12 \mu$ sec it has spread azimuthally as well. Typical discharge voltage, current and plasma flux are shown in Fig. 18. The discharge voltage is low indicating that a much larger current can be supported before onset (characterized by a $V_{d}$ of typically several hundred volts). The plasma flow velocity can be crudely obtained by the time difference between the peak discharge current, $I_{d}$ and the peak flux ion flux, $\Gamma_{\mathrm{i}}$. With the probe $15 \mathrm{~cm}$ downstream of the plasma anode, a flow

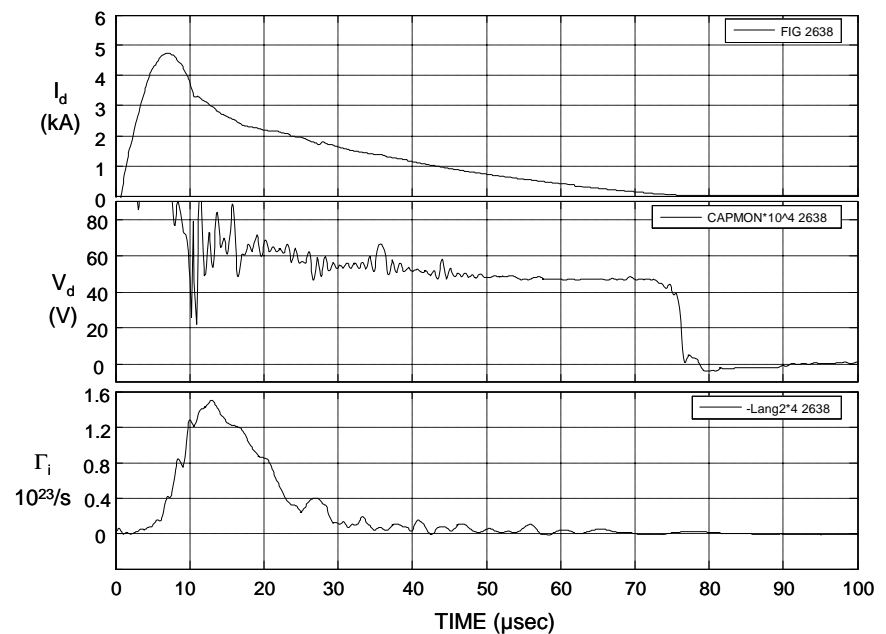

Figure 18. Discharge voltage, current and plasma signal from a mini-MPD source. velocity of $2.5 \mathrm{~cm} / \mu \mathrm{sec}$ or 25 $\mathrm{km} / \mathrm{sec}$ is inferred. This is typical of an MPD operating near the critical speed for Deuterium. 


\section{Results of 2D MHD calculations.}

In addition to the modifications to the IPA-C magnetic compression coils and energy storage modules, the new formation coils have been installed and tested. Full operation is scheduled for the March-April 08 timeframe. 2D MHD numerical calculations were carried out for the IPA-C experiment. The timing sequences and magnetic fields for formation, acceleration, merging and compression were input into these calculations based on the expected values. The results are displayed in Fig. 19. The evolution of the key plasma parameters can be found in Fig. 20.
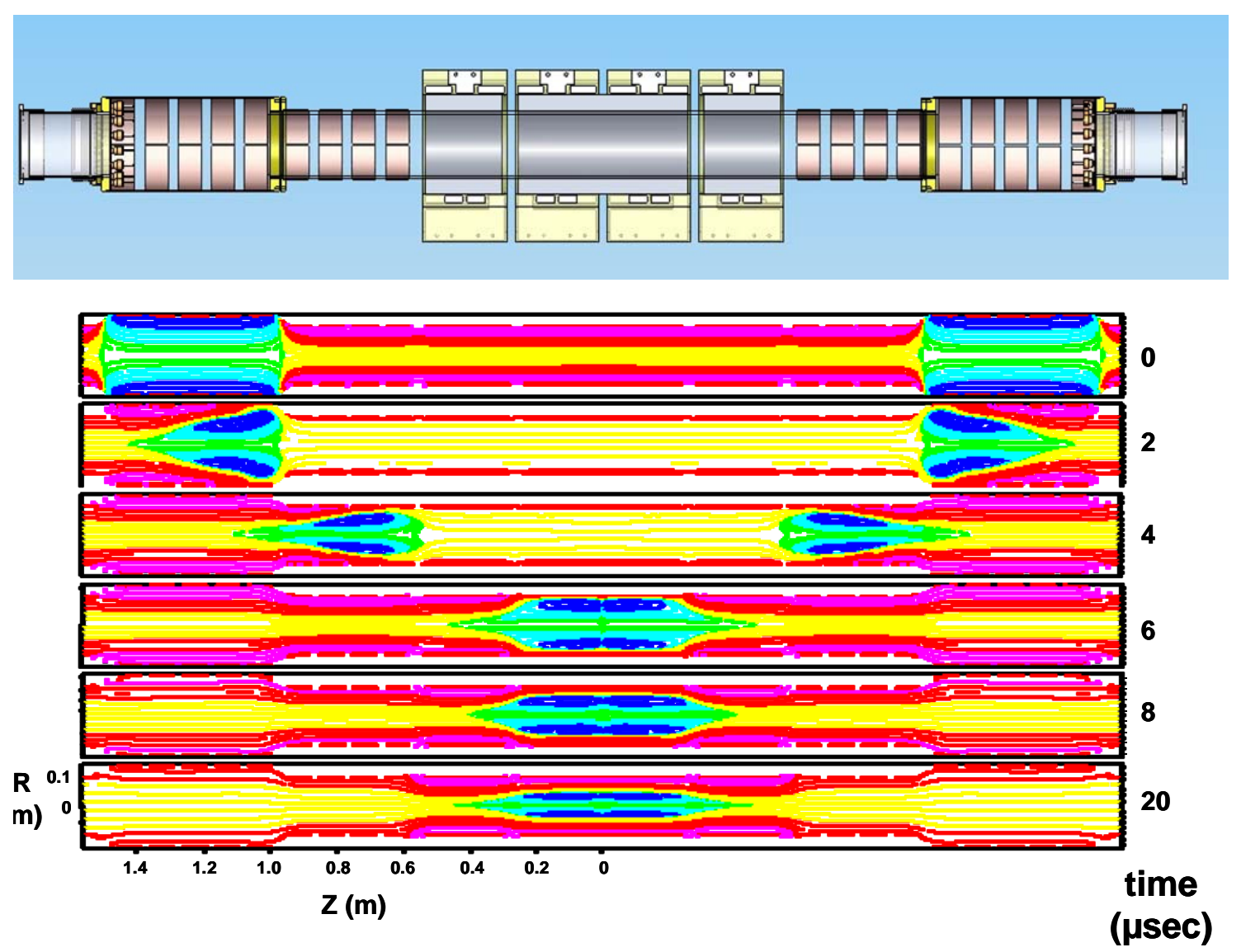

Figure 19. MHD Calculation for the current IPA-C experiment

From these figures the rapid conversion of plasma directional energy into ion thermal energy at $\sim 6 \mu \mathrm{sec}$ is clearly observed. Both the plasma ion and electron temperature increase adiabatically during the magnetic compression that ensues after the plasmoid merging, and the merging energy input is almost entirely into the ions.. In previous experiments on IPA-C the maximum field was $\sim 0.8 \mathrm{~T}$ but plasma temperatures $\sim 1 \mathrm{keV}$ were achieved. The current upgrade will bring the ion temperature into a range where there should be significantly enhanced neutron production (see Fig. 10). Two new calibrated neutron detectors will also be installed to confirm this. The anticipated neutron flux for a D-T mix can be calculated from Eq. (10) and Fig. 10 where: 


$$
\mathrm{B}=2 \mathrm{~T}, \quad \mathrm{~T}_{\mathrm{i}}=3 \mathrm{keV} \quad \mathrm{E}_{\mathrm{p}}=10 \mathrm{~kJ} \quad \varphi_{\mathrm{p}}=2 \mathrm{mWb} \quad \mathrm{r}_{\mathrm{c}}=0.10 \mathrm{~m} .
$$

The result is

$$
\mathrm{N}_{\text {neut }}=2.62 \times 10^{31} \frac{2^{7 / 4}\left(10^{4}\right)^{3 / 2} \sqrt{0.002}}{0.10^{1 / 3}} 10^{-25}=8.5 \times 10^{11}
$$

This is close to what is expected from the MTF experiments to be conducted at AFRL.

The work described in this report is part of the collaborative effort on the Magnetized Target Fusion (MTF) experiments being conducted at the Los Alamos National Laboratory (LANL) entitled "Physics Demonstration for Magnetized Target Fusion via Metal Liner Implosion of a Field-Reversed Configuration Plasma". The objective of the work is to design, construct, test, and help operate a FRC formation coilset at Los Alamos that can achieve the parameters required for the target FRC plasmas to be imploded. The focus of the work performed in the second year has been enhanced flux trapping, as this now seems to
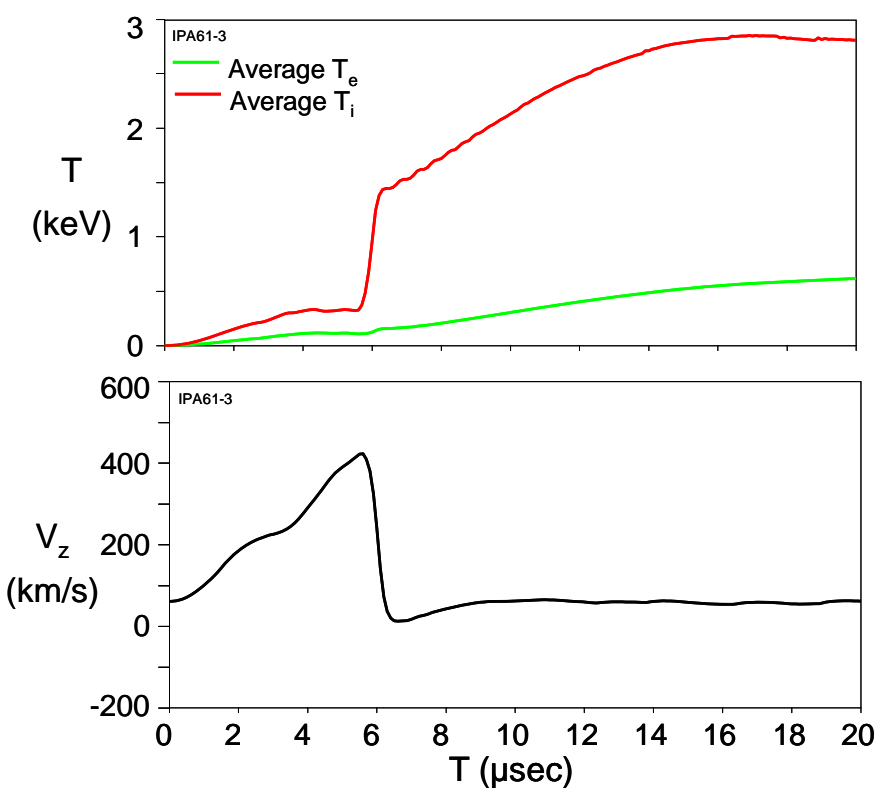

Figure 20. Evolution of the plasma temperature and FRC velocity during the formation, acceleration, merging and compression of two FRC plasmoids in IPA-C.

be the major remaining issue in achieving the target parameters desired for the FRC. It has been clear that given the budget constraints, solutions to this problem must be done in a manner that requires as little modification to the existing theta pinch hardware as possible.

Based on previous work by the PI, the achievement of the equilibrium flux $\phi_{\text {eq }}$ and confinement time $\tau$ required for the target FRC ( $\left.\phi_{\text {eq }} \sim 1 \mathrm{mWb}, \tau>40 \mu \mathrm{s}\right)$ can only be attained by employing the proper formation techniques. The use of enhanced programmed formation was found essential to improve the symmetry, reduce impurity concentration and maintain flux retention of the FRC through formation at high bias in previous experiments. This method of formation has been adopted by LANL and has resulted in the formation of long-lived, MHD stable FRCs. The preionization technique used in these experiments was also adopted from that found successful on the LSX experiments where a slow ringing theta preionization was employed. The slower oscillation at first seems to be the opposite of what would be desired to rapidly and 
thoroughly ionize the initial gas. What was found on LSX, and repeated in the LANL experiments, is that the ionization is mainly attributable to the enhanced electron mobility in the azimuthal electric field at the zero crossing of the axial magnetic field. A slow rate change of the field increased the ionization time, as well as minimized large scale motion of the plasma. The lift-off flux at field reversal (flux left inside the vacuum chamber) is very limited with this preionization scheme however. It is due to the fact that one must diffuse all of the bias (reverse) field back into the preionized plasma in order to trap it. The effort at PDL has been to avoid the use of this preionization technique, and perform the preionization in a new and different way - a way that permits nearly all of the bias field to be inside the plasma sheath at reversal.

The use of some supplementary discharge preionization will be necessary to achieve the required initial trapped reverse flux. As mentioned, the changes required to the FRXL experiment to be accomplished without significant modification of the existing facility. The design, construction and testing of the additional barrier magnet system and discharge preionization system, which has proven to improve the trapped flux in past experiments at MSNW and the UW, was to be the first approach to this problem. The modifications and costs required to install the necessary systems was deemed too be to large. This necessitated a change in the approach. There were other preionization schemes that did not rely on modulation of the magnetic field, such as axial discharge preionization. This also required internal electrode structures that would make the translation more difficult, as well as increased the electrical difficulties, as the FRX-L coil set is not insulated for high voltage.

Where there is a will, there is a way. There are two promising new approaches that have been formulated by the Plasma Dynamics Laboratory (PDL) at the University of Washington to solve this problem. The first was the application of a Rotating Magnetic Field (RMF) for edge preionization. This approach was tested at PDL on the Pulsed High Density Experiment (PHD). These experiments were quite successful and the appropriate coil set for MTF is being designed.

The second approach takes advantage of the expertise at PDL in high power electric propulsion. In this approach, a modified Magneto-PlasmaDynamic (MPD) thruster is used for preionization. The neutral Deuterium gas is ionized and injected along the axial field lines at the appropriately high fluence to provide of essentially $100 \%$ preionization inside the FRX-L formation coil. A more detailed discussion of these two approaches will be now be given.

During Field Reversal:

-Counter-rotate ions $\Rightarrow$ increase FRC stable period

-Increase lift-off flux in FRC

-Reduce impurity pick-up

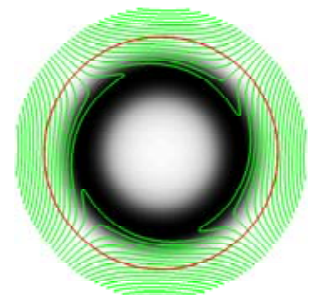

\section{Equilibrium:}

-Provide rotational stabilization

-Improve particle confinement

Figure 1. Rotating barrier field concept 


\section{RMF Preionization Studies}

The focus of the parameter improvements on FRX-L has also shifted to methods that could solve more than just the formation problems. These new approaches had to afit in the framework of being able to be retrofitted to the existing device as well as any future modification required for the source to be constructed for the liner experiments. The changes took the form of a different barrier field configuration that could both pre-ionize and stabilize the FRC. The new approach is illustrated in Fig. 1. The several major contributions that this one system can provide are iterated in this figure. This system has now been tested and proven to provide adequate flux trapping on the PHD experiment. The use of the rotating barrier field (RBF) is still a matter of some concern as it will require a change in the coil opacity. It will also require coils to be installed external to the FRX-L theta pinch coils so that space and insulation should not be major issues. A schematic of the RBF coils as used on the PHD experiment is shown in Fig. 2. As can be seen, the coils can be mounted external to the theta pinch coils, and be compatible with high voltage. The transparency of the PHD accelerator coils is quite high $\sim 80 \%$. It is not believed that it need be anywhere near this high to be effective. Successful RMF experiments were conducted in a test setup for the TCS experiments with a coil transparency of less than $40 \%$.

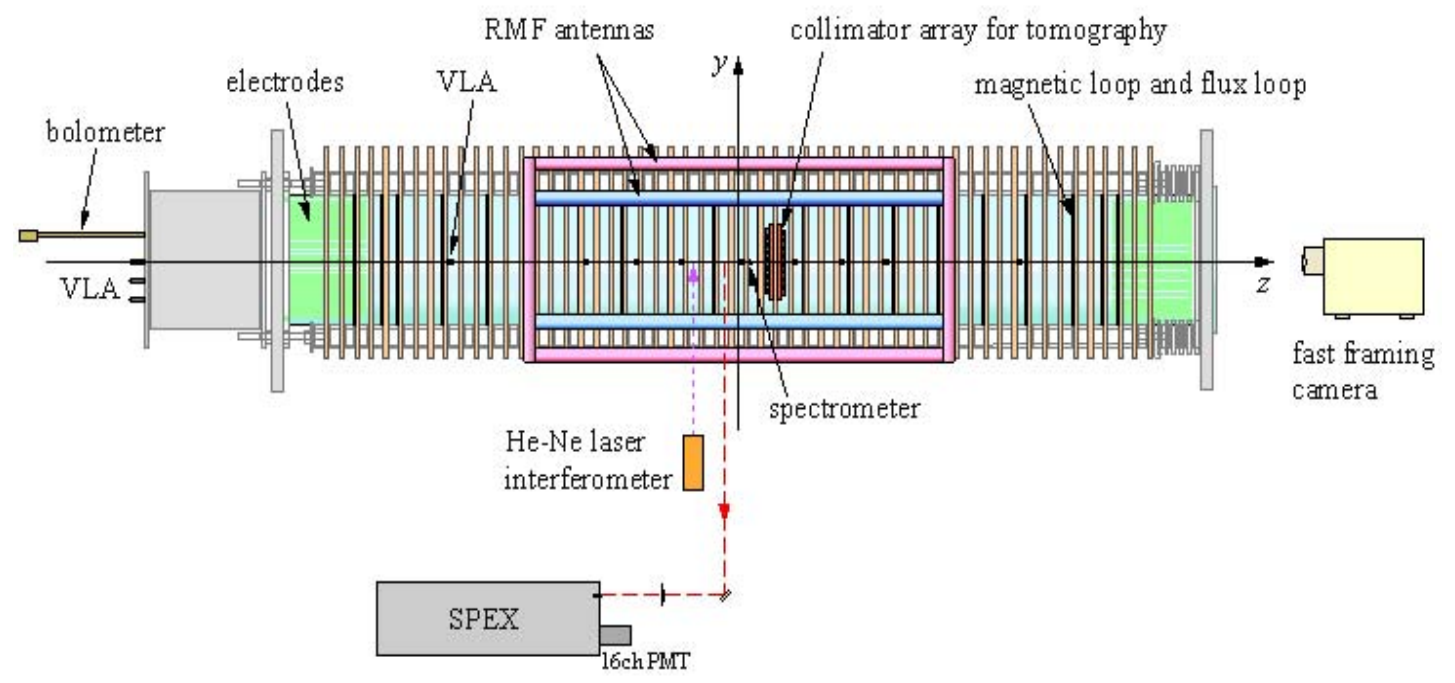

Figure 2. Test setup for flux trapping studies on PHD with RBF

The RMF system by itself can not be used to form the FRC as the energy that is available for FRC formation is too small by many orders of magnitude. Typically, one assumes roughly $50 \mathrm{eV}$ per ionization of the Deuterium atom, and for the target FRX-L inventory of $5 \times 10^{19}$ ions, the ionization energy is $400 \mathrm{~J}$. The timescale for ionization is roughly the transit time for the preionized plasma to flow out axially, which for $\mathrm{T} \sim 2 \mathrm{eV}$ and FRX-L $\left(\mathrm{L}_{\mathrm{c}} \sim 0.3 \mathrm{~m}\right)$ is $30 \mu \mathrm{sec}$. The power flow is thus $13 \mathrm{MW}$. It is actually possible to provide this level of power input, and it has been achieved in recent experiments on PHD with pulsed oscillators. Due to the much larger scale of the PHD 
source $\left(\mathrm{r}_{\mathrm{c}}=0.2 \mathrm{~m}\right.$ for PHD vs $0.05 \mathrm{~m}$ for FRX-L), the fractional ionization was much lower.

\section{MPD Plasma Source}

In addition to the rotating barrier approach, an end-on preionization scheme that does not require internal electrodes within the formation region is being designed and constructed. This method of providing the plasma required for the FRC is based on the MPD thruster physics developed for electric propulsion. The plasma mass flow required to fill the $0.3 \mathrm{~m}$ long source in $30 \mu \mathrm{sec}$ to full preionization requires a flow velocity of only $1 \times 10^{4} \mathrm{~m} / \mathrm{s}$, which is easily achieved in the MPD thruster. For the equivalent density of a 75 mTorr $D_{2}$ fill, $\left(10^{19} \mathrm{D}\right.$ ions) the mass flow is $1.25 \mathrm{~g} / \mathrm{sec}$. MPD thrusters can supply plasma at a rate of 3 to $6 \mathrm{~g} / \mathrm{sec}$, so the device should provide full ionization and the maximum possible flux trapping as there will be no need for the ringing theta PI. The prototype device bein constructed is shon in Fig. 3. If the MPD works as expected, the operational flux and fill density can be significantly increased, and bring the FRX-L plasma density and flux into the target range. Since the plasma created in the MPD source propagates across and down the axial bias magnetic field lines, the source can be located upstream of the high voltage theta pinch coils, and provide for a simple way to maintain the vacuum in both the translation and liner sections.

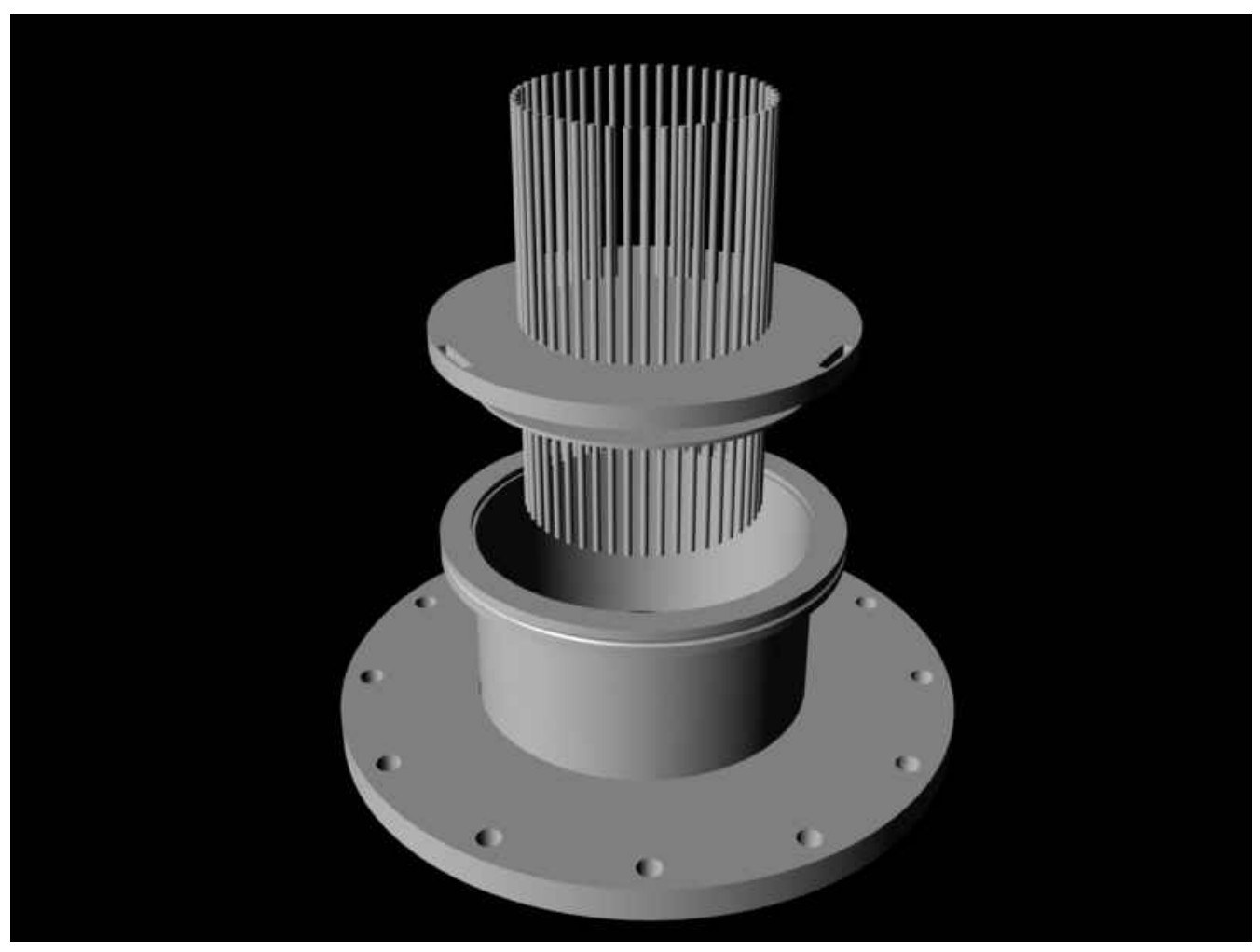

Figure 3. MPD plasma source tungsten electrode wires in stainless steel vacuum anode.

The construction and testing of these other approaches occupied most of the work performed at the UW. 


\section{Summary}

Nuclear fusion has the potential to satisfy the prodigious power that the world will demand in the future, but it has yet to be harnessed as a practical energy source. The entry of fusion as a viable, competitive source of power has been stymied by the challenge of finding an economical way to provide for the confinement and heating of the plasma fuel. It is the contention here that a simpler path to fusion can be achieved by creating fusion conditions in a different regime at small scale $(\sim$ a few $\mathrm{cm})$. One such program now under study, referred to as Magnetized Target Fusion (MTF), is directed at obtaining fusion in this high energy density regime by rapidly compressing a compact toroidal plasmoid commonly referred to as a Field Reversed Configuration (FRC). To make fusion practical at this smaller scale, an efficient method for compressing the FRC to fusion gain conditions is required. In one variant of MTF a conducting metal shell is imploded electrically. This radially compresses and heats the FRC plasmoid to fusion conditions. The closed magnetic field in the target plasmoid suppresses the thermal transport to the confining shell, thus lowering the imploding power needed to compress the target.

The undertaking described in this report was to provide a suitable target FRC, as well as a simple and robust method for inserting and stopping the FRC within the imploding liner. The FRC must also survive during the time it takes for the metal liner to compress the FRC target. The initial work a the UW was focused on developing adequate preionization and flux trapping that were found to be essential in past experiments for obtaining the density, flux and most critically, FRC lifetime required for MTF.

The timescale for testing and development of such a source can be rapidly accelerated by taking advantage of a new facility funded by the Department of Energy. At this facility, two inductive plasma accelerators (IPA) were constructed and tested. Recent experiments with these IPAs have demonstrated the ability to rapidly form, accelerate and merge two hypervelocity FRCs into a compression chamber. The resultant FRC that was formed was hot ( $\mathrm{T}_{\text {ion }} \sim 400 \mathrm{eV}$ ), stationary, and stable with a configuration lifetime several times that necessary for the MTF liner experiments. The accelerator length was less than 1 meter, and the time from the initiation of formation to the establishment of the final equilibrium was less than 10 microseconds.

With some modification, each accelerator can be made capable of producing FRCs suitable for the production of the target plasma for the MTF liner experiment. Based on the initial FRC merging/compression results, the design and methodology for an experimental realization of the target plasma for the MTF liner experiment can now be defined. The construction and testing of the key components for the formation of the target plasma at the Air force Research Laboratory (AFRL) will be performed on the IPA experiment, now at MSNW. A high density FRC plasmoid will be formed and accelerated out of each IPA into a merging/compression chamber similar to the imploding liner at AFRL. The properties of the resultant FRC plasma (size, temperature, density, flux, lifetime) will be obtained. The process will be optimized, and a final design for implementation at AFRL will be carried out. When implemented at AFRL it is 
anticipated that the colliding/merging FRCs will then be compressed by the liner. In this manner it is hoped that ultimately a plasma with ion temperatures reaching the $10 \mathrm{keV}$ range and fusion gain near unity can be obtained. 